\title{
UNIQUENESS OF UNCONDITIONAL BASES IN BANACH SPACES
}

\author{
P.G. CASAzZa And N.J. Kalton
}

\begin{abstract}
.
We prove a general result on complemented unconditional basic sequences in Banach lattices and apply it to give some new examples of spaces with unique unconditional basis. We show that Tsirelson space and certain Nakano spaces have unique unconditional bases. We also construct an example of a space with a unique unconditional basis with a complemented subspace failing to have a unique unconditional basis.
\end{abstract}

\section{InTRODUCTION}

A Banach space with an unconditional basis is said to have a unique unconditional basis if any two normalized unconditional bases are equivalent after a permutation. It is well-known that $\ell_{2}$ has a unique unconditional basis (cf. [17]) and a classic result of Lindenstrauss and Pełczynski [18] asserts that the spaces $\ell_{1}$ and $c_{0}$ also have unique unconditional bases; later Lindenstrauss and Zippin [21] showed that this is the complete list of spaces with symmetric bases for which the unconditional basis is unique. Subsequently Edelstein and Wojtaszczyk [10] showed that direct sums of $\ell_{1}, \ell_{2}$ and $c_{0}$ also have unique unconditional bases. In 1985, Bourgain, Casazza, Lindenstrauss and Tzafriri [3] studied the classification problem for such spaces. Their main results showed that $\ell_{1}\left(\ell_{2}\right), c_{0}\left(\ell_{1}\right), \ell_{1}\left(c_{0}\right), c_{0}\left(\ell_{2}\right)$ and 2-convexified Tsirelson $T^{(2)}$ have unique unconditional bases but that $\ell_{2}\left(\ell_{1}\right)$ and $\ell_{2}\left(c_{0}\right)$ do not. Based on their results a complete classification looks hopeless. We also remark that a recent example of Gowers [12] may be easily shown to have unique unconditional basis. Thus there are many "pathological" spaces with unique unconditional basis.

1991 Mathematics Subject Classification. 46B15, 46B07.

Both authors were supported by NSF Grant DMS-9201357 
In this paper we will give (Theorem 3.5) a simple and, we feel, useful characterization of complemented unconditional basic sequences in Banach sequence spaces which are not sufficiently Euclidean (i.e. do not have uniformly complemented $\ell_{2}^{n}$ 's). This theorem is the discrete analogue of Theorem 8.1 of [14]; in fact the basic arguments are very similar to those given in [16] and [14], but we have opted to present a self-contained proof here. We then use this result and the recent work of Wojtaszczyk [26] to give some more examples of fairly natural spaces with unique unconditional basis. In Section 5, we introduce the class of left- and right-dominant bases and use this notion to show that the Nakano space $\ell\left(p_{n}\right)$ has a unique unconditional basis if $p_{n} \downarrow 1$ and $\left(p_{n}-p_{2 n}\right) \log n$ is bounded (there is a dual result if $p_{n} \uparrow \infty$ ). We also show that Tsirelson space $T$ has a unique unconditional basis (a question raised in [3] p. 62). In Section 6, we use similar techniques to show that certain complemented subspaces of Orlicz sequence spaces have unique unconditional bases. Based on these examples we are able to resolve Problem 11.2 (p.104) of [3] by showing that there is a space with unique unconditional basis with a complemented subspace (spanned by a subsequence of the basis) failing to have unique unconditional basis.

Also in Section 4, we use Theorem 3.5 to give a contribution to the problem of uniqueness of unconditional bases in finite-dimensional spaces. Specifically, we prove that in any class of finite-dimensional lattices so that $\ell_{2}^{n}$ is not complementably and disjointly representable, the unconditional basis is almost unique; for a more precise statement see Theorem 4.1 .

We remark that the techniques developed here using Theorem 3.5 can be used successfully to obtain other results on uniqueness. In particular we plan to study unconditional bases in $c_{0}$-products in a later publication. Since the arguments in such spaces are considerably more complicated, it seemed, however, appropriate to restrict attention here to some simple applications.

\section{Definitions and notation}

We will take the viewpoint that an unconditional basis in a Banach space $X$ confers the structure of an atomic Banach lattice on $X$. We will thus adopt the language and structure of Banach lattices. It is well-known that a separable Banach lattice can be regarded as a Köthe function space.

We will in general use the same notation as in [16]. Let $\Omega$ be a Polish space (i.e. a separable complete metric space) and let $\mu$ be a $\sigma$-finite Borel measure on 
$\Omega$. We denote by $L_{0}(\mu)$ the space of all Borel measurable functions on $\Omega$, where we identify functions differing only on a set of measure zero; the natural topology of $L_{0}$ is convergence in measure on sets of finite measure. An admissible norm is then a lower-semi-continuous map $f \rightarrow\|f\|$ from $L_{0}(\mu)$ to $[0, \infty]$ such that:

(a) $\|\alpha f\|=|\alpha|\|f\|$ whenever $\alpha \in \mathbf{R}, f \in L_{0}$.

(b) $\|f+g\| \leq\|f\|+\|g\|$, for $f, g \in L_{0}$.

(c) $\|f\| \leq\|g\|$, whenever $|f| \leq|g|$ a.e. (almost everywhere).

(d) $\|f\|<\infty$ for a dense set of $f \in L_{0}$,

(e) $\|f\|=0$ if and only if $f=0$ a.e.

A Köthe function space on $(\Omega, \mu)$ is defined to be a dense order-ideal $X$ in $L_{0}(\mu)$ with an associated admissible norm \|\|$_{X}$ such that if $X_{\max }=\left\{f:\|f\|_{X}<\infty\right\}$ then either:

(1) $X=X_{\max }$ ( $X$ is maximal) or:

(2) $X$ is the closure of the simple functions in $X_{\max }$ ( $X$ is minimal).

Any order-continuous Köthe function space is minimal. Also any Köthe function space which does not contain a copy of $c_{0}$ is both maximal and minimal.

If $X$ is an order-continuous Köthe function space then $X^{*}$ can be identified with the Köthe function space of all $f$ such that:

$$
\|f\|_{X^{*}}=\sup _{\|g\|_{X} \leq 1} \int|f g| d \mu<\infty .
$$

$X^{*}$ is always maximal.

A Köthe function space $X$ is said to be $p$-convex (where $1<p<\infty$ ) if there is a constant $C$ such that for any $f_{1}, \ldots, f_{n} \in X$ we have

$$
\left\|\left(\sum_{i=1}^{n}\left|f_{i}\right|^{p}\right)^{1 / p}\right\|_{X} \leq C\left(\sum_{i=1}^{n}\left\|f_{i}\right\|_{X}^{p}\right)^{1 / p} .
$$

$X$ is said to have an upper $p$-estimate if for some $C$ and any disjoint $f_{1}, \ldots, f_{n} \in$ $X$,

$$
\left\|\sum_{i=1}^{n} f_{i}\right\|_{X} \leq C\left(\sum_{i=1}^{n}\left\|f_{i}\right\|_{X}^{p}\right)^{1 / p} .
$$

$X$ is said to be $q$-concave $(0<q<\infty)$ if for some $c>0$ and any $f_{1}, \ldots, f_{n} \in X$ we have

$$
\left\|\left(\sum_{i=1}^{n}\left|f_{i}\right|^{q}\right)^{1 / q}\right\|_{X} \geq c\left(\sum_{i=1}^{n}\left\|f_{i}\right\|_{X}^{q}\right)^{1 / q} .
$$


$X$ is said to have a lower $q$-estimate if for some $c>0$ and any disjoint $f_{1}, \ldots, f_{n} \in$ $X$,

$$
\left\|\sum_{i=1}^{n} f_{i}\right\|_{X} \geq c\left(\sum_{i=1}^{n}\left\|f_{i}\right\|_{X}^{q}\right)^{1 / q} .
$$

A Banach space $X$ is said to be of (Rademacher) type $p(1 \leq p \leq 2)$ if there is a constant $C$ so that for any $x_{1}, \ldots, x_{n} \in X$,

$$
\underset{\epsilon_{i}= \pm 1}{\operatorname{Ave}}\left\|\sum_{i=1}^{n} \epsilon_{i} x_{i}\right\| \leq C\left(\sum_{i=1}^{n}\left\|x_{i}\right\|^{p}\right)^{1 / p}
$$

and $X$ is of cotype $q(2 \leq q<\infty)$ if for some $c>0$ and any $x_{1}, \ldots, x_{n} \in X$ we have

$$
\underset{\epsilon_{i}= \pm 1}{\operatorname{Ave}}\left\|\sum_{i=1}^{n} \epsilon_{i} x_{i}\right\| \geq c\left(\sum_{i=1}^{n}\left\|x_{i}\right\|^{q}\right)^{1 / q} .
$$

We recall that a Banach lattice has nontrivial cotype (i.e. has cotype $q<\infty$ for some $q$ ) if and only if it has nontrivial concavity (i.e. is $q$-concave for some $q<\infty)$. If $X$ is a Banach lattice which has nontrivial concavity then there is a constant $C=C(X)$ so that for any $x_{1}, \ldots, x_{n} \in X$ we have

$$
\frac{1}{C}\left({\underset{\epsilon}{\epsilon}= \pm 1}_{k=1}\left\|\sum_{k=1}^{n} \epsilon_{k} x_{k}\right\|^{2}\right)^{1 / 2} \leq\left\|\left(\sum_{k=1}^{n}\left|x_{k}\right|^{2}\right)^{1 / 2}\right\|_{X} \leq C\left(\underset{\epsilon_{k}= \pm 1}{\text { Ave }}\left\|\sum_{k=1}^{n} \epsilon_{k} x_{k}\right\|^{2}\right)^{1 / 2} .
$$

We will use the term sequence space to denote a Köthe function space $X$ on $\mathbf{N}$ equipped with counting measure, and subject to the normalization constraint that $\left\|e_{j}\right\|_{X}=1$ for all $j \in \mathbf{N}$ where $e_{j}=\chi_{\{j\}}$. It is clear that $\left(e_{n}\right)$ forms an unconditional basis for a sequence space $X$ if and only if $X$ is minimal (or separable). We will consider finite-dimensional sequence spaces modelled on finite sets $[N]=\{1,2, \ldots, N\}$ with counting measure.

In keeping with current usage we will write $c_{00}$ for the space of finitely nonzero sequences. If $A$ is a subset of $\mathbf{N}$ we write $e_{A}$ in place of $\chi_{A}$ and if $x$ is any sequence we write $A x=e_{A} x$. If $A, B$ are subsets of $\mathbf{N}$ we write $A<B$ if $a<b$ whenever $a \in A$ and $b \in B$. If $x$ is a sequence then supp $x=\{i: x(i) \neq 0\}$.

Many of our examples will be Orlicz sequence spaces or more general OrliczMusielak or modular sequence spaces. If $\left(F_{n}\right)$ is a sequence of Orlicz functions then the modular sequence space $\ell_{\left(F_{n}\right)}$ is the space of sequences $(x(n))_{n=1}^{\infty}$ such that $\sum_{n=1}^{\infty} F_{n}(|x(n)|)<\infty$, with the norm

$$
\|x\|_{\ell\left(F_{n}\right)}=\inf \left\{\lambda>0: \sum_{n=1}^{\infty} F_{n}\left(\lambda^{-1}|x(n)|\right) \leq 1\right\} .
$$


In the case $F_{n}=F$ for all $n$ we have the Orlicz space $\ell_{F}$. If $\ell_{\left(F_{n}\right)}$ is separable or has finite cotype then the canonical basis vectors form an unconditional basis of $\ell_{\left(F_{n}\right)}$; otherwise they form an unconditional basis of their closed linear span $h_{\left(F_{n}\right)}$. We refer to [19] for the basic properties of modular sequence spaces.

One special case is to take $F_{n}(t)=t^{p_{n}}$ where $1 \leq p_{n}<\infty$. This is often called a Nakano space and we denote it $\ell\left(p_{n}\right) \cdot \ell\left(p_{n}\right)$ is separable if and only if $\sup p_{n}<\infty$. It may also be shown that if $p_{n}>1$ for all $n$ and $\sup p_{n}<\infty$ then $\ell\left(p_{n}\right)^{*}=\ell\left(q_{n}\right)$ where $p_{n}^{-1}+q_{n}^{-1}=1$. If $\sup p_{n}=\infty$ then we write $h\left(p_{n}\right)$ for the closed linear span of the basis vectors, and we have $h\left(p_{n}\right)^{*}=\ell\left(q_{n}\right)$.

Let $\left(u_{n}\right)$ and $\left(v_{n}\right)$ be two unconditional basic sequences. We say that $\left(u_{n}\right)$ and $\left(v_{n}\right)$ are permutatively equivalent if there is a permutation $\pi$ of $\mathbf{N}$ so that $\left(u_{n}\right)$ and $\left(v_{\pi(n)}\right)$ are equivalent. We say that $\left(u_{n}\right)$ is equivalent to its square if $\left(u_{n}\right)$ is permutatively equivalent to the basis $\left\{\left(u_{1}, 0\right),\left(0, u_{1}\right),\left(u_{2}, 0\right), \ldots\right\}$ of $\left[u_{n}\right] \oplus\left[u_{n}\right]$. A Banach space $X$ with an unconditional basis has a unique unconditional basis if any two normalized unconditional bases are permutatively equivalent. We remark that there is an important Cantor-Bernstein type principle which helps determine whether two unconditional bases are permutatively equivalent: if $\left(u_{n}\right)$ is permutatively equivalent to some subset of $\left(v_{n}\right)$ and if $\left(v_{n}\right)$ is permutatively equivalent to some subset of $\left(u_{n}\right)$ then $\left(u_{n}\right)$ and $\left(v_{n}\right)$ are permutatively equivalent. We are grateful to P. Wojtaszczyk for drawing our attention to this principle, which appears explicitly in [27] and is used in [26]. We are indebted to C. Bessaga for the information that the Cantor-Bernstein principle was used implicitly earlier by Mityagin in [22].

A Banach space $X$ is called sufficiently Euclidean if there is a constant $M$ so that for any $n$ there are operators $S: X \rightarrow \ell_{2}^{n}$ and $T: \ell_{2}^{n} \rightarrow X$ so that $S T=I_{\ell_{2}^{n}}$ and $\|S\|\|T\| \leq M$. We will say that $X$ is anti-Euclidean if it is not sufficiently Euclidean.

A Banach lattice $X$ is called sufficiently lattice Euclidean if there is a constant $M$ so that for any $n$ there are operators $S: X \rightarrow \ell_{2}^{n}$ and $T: \ell_{2}^{n} \rightarrow X$ so that $S T=I_{\ell_{2}^{n}}$ and $\|S\|\|T\| \leq M$, and such that $S$ is a lattice homomorphism. This is equivalent to asking that $\ell_{2}$ is finitely representable as a complemented sublattice of $X$. We will say that $X$ is lattice anti-Euclidean if it is not sufficiently lattice Euclidean. We use the same terminology for an unconditional basic sequence, which we regard as inducing a lattice structure on its closed linear span.

Finally if $\mathcal{X}$ is a family of Banach lattices we say that $\mathcal{X}$ is sufficiently lattice 
Euclidean if there is a constant $M$ so that for any $n$ there exists $X \in \mathcal{X}$ and operators $S: X \rightarrow \ell_{2}^{n}$ and $T: \ell_{2}^{n} \rightarrow X$ so that $S T=I_{\ell_{2}^{n}}$ and $\|S\|\|T\| \leq M$, and such that $S$ is a lattice homomorphism. If $\mathcal{X}$ is not sufficiently lattice Euclidean we will say that it is lattice anti-Euclidean.

\section{Complemented UnCONDitional BASIC SEQUENCES}

The main results of this section are Theorems 3.4 and 3.5, which show that complemented lattice anti-Euclidean unconditional basic sequences in an ordercontinuous Banach lattice or Banach sequence space take a particularly simple form.

Lemma 3.1. Let $X$ be a Banach sequence space and suppose $\left(u_{1}, \ldots, u_{n}\right)$ are disjoint elements of $X_{+}$, and $\left(u_{1}^{*}, \ldots, u_{n}^{*}\right)$ are disjoint in $X_{+}^{*}$. Suppose that $M \geq$ 1 is a constant such that

$$
\left\|\sum_{j=1}^{n} a_{j} u_{j}\right\|_{X} \leq M\left(\sum_{j=1}^{n}\left|a_{j}\right|^{2}\right)^{1 / 2}
$$

and

$$
\left\|\sum_{j=1}^{n} a_{j} u_{j}^{*}\right\|_{X^{*}} \leq M\left(\sum_{j=1}^{n}\left|a_{j}\right|^{2}\right)^{1 / 2}
$$

whenever $a_{1}, \ldots, a_{n} \in \mathbf{R}$. Suppose further that

$$
\sum_{j=1}^{n}\left\langle u_{j}, u_{j}^{*}\right\rangle=\alpha n
$$

Then $\ell_{2}^{m}$ is $2 M^{2} \alpha^{-1}$-representable as a $2 M^{2} \alpha^{-1}$-complemented sublattice of $X$, for some $m \geq \frac{1}{2} \alpha M^{-2} n$.

Proof. We can clearly suppose that $\operatorname{supp} u=\operatorname{supp} u^{*}$. Note that $\left\langle u_{i}, u_{i}^{*}\right\rangle \leq M^{2}$. Let $J=\left\{j:\left\langle u_{j}, u_{j}^{*}\right\rangle \geq \frac{1}{2} \alpha\right\}$. Then $|J| \geq \frac{1}{2} M^{-2} \alpha n$. Notice that for any $\left(a_{j}\right)_{j \in J}$ we have

$$
\left\|\sum_{j \in J} a_{j} u_{j}\right\|_{X}\left\|\sum_{j \in J} a_{j} u_{j}^{*}\right\|_{X^{*}} \geq \frac{\alpha}{2}\left(\sum_{j \in J}\left|a_{j}\right|^{2}\right) .
$$

Thus

$$
\frac{\alpha}{2 M}\left(\sum_{j \in J}\left|a_{j}\right|^{2}\right)^{1 / 2} \leq\left\|\sum_{j \in J} a_{j} u_{j}\right\|_{X} \leq M\left(\sum_{j \in J}\left|a_{j}\right|^{2}\right)^{1 / 2}
$$


so that $\left[u_{j}\right]_{j \in J}$ is $\left(2 M^{2}\right) / \alpha$-lattice isomorphic to $\ell_{2}^{|J|}$. If we let $\gamma_{j}=\left\langle u_{j}, u_{j}^{*}\right\rangle^{-1}$ then we can define a projection $P$ onto $\left[u_{j}\right]_{j \in J}$ by

$$
P x=\sum_{j \in J} \gamma_{j}^{-1}\left\langle x, u_{j}^{*}\right\rangle u_{j}
$$

Then

$$
\|P x\|_{X} \leq \frac{2 M}{\alpha}\left(\sum_{j \in J}\left|\left\langle x, u_{j}^{*}\right\rangle\right|^{2}\right)^{1 / 2}
$$

However,

$$
\begin{aligned}
\sum_{j \in J}\left|\left\langle x, u_{j}^{*}\right\rangle\right|^{2} & =\left\langle x, \sum_{j \in J}\left\langle x, u_{j}^{*}\right\rangle u_{j}^{*}\right\rangle \\
& \leq M\|x\|_{X}\left(\sum_{j \in J}\left\langle x, u_{j}^{*}\right\rangle^{2}\right)^{1 / 2}
\end{aligned}
$$

whence we obtain

$$
\|P x\|_{X} \leq \frac{2 M^{2}}{\alpha}\|x\|_{X}
$$

Lemma 3.2. Let $X$ be a order-continuous Köthe function space on $(\Omega, \mu)$. Suppose $m \in \mathbf{N}$, and $\phi \in L_{1}(\mu)$ with $\phi \geq 0$. Suppose $f_{1}, \ldots, f_{n} \in X_{+}$and let $F=\left(\sum_{j=1}^{n} f_{j}^{2}\right)^{1 / 2}$ and $F_{\infty}=\max _{j} f_{j}$. Then we can partition $[n]=\{1,2, \ldots, n\}$ into $m$-sets $J_{1}, \ldots, J_{m}$ and find a set $A \subset \Omega$ with $\int_{A} \phi d \mu \geq \frac{3}{4} \int_{\Omega} \phi d \mu$ so that whenever $a_{1}, \ldots, a_{m} \in \mathbf{R}$ we have

$$
\left\|\left(\sum_{k=1}^{m} a_{k}^{2} \sum_{j \in J_{k}} f_{j}^{2}\right)^{1 / 2} \chi_{A}\right\|_{X} \leq 2\left(\|F\|_{X}+2.5^{m}\left\|F_{\infty}\right\|_{X}\right) m^{-1 / 2}\left(\sum_{k=1}^{m} a_{k}^{2}\right)^{1 / 2} .
$$

Proof. We may select a collection of $5^{m}$ points $\left(b^{r}\right)_{r=1}^{5^{m}}$ in the unit sphere of $\ell_{2}^{m}$ to form a $\frac{1}{2}$-net. Then if $T: \ell_{2}^{m} \rightarrow Y$ is any operator we have $\|T\| \leq$ $2 \sup _{r \leq 5^{m}}\left\|T b^{r}\right\|_{Y}$.

Next let $\Pi$ be the set of all $m^{n}$ partitions of $[n]$ into $m$-sets $\pi=\left(J_{1}, \ldots, J_{m}\right)$ with a probability measure $P$ defined to be normalized counting measure. For $1 \leq l \leq m$ and $1 \leq j \leq n$ we define $\xi_{l j}(\pi)=1$ if $j \in J_{l}$ and 0 otherwise. The random variables $\xi_{k i}, \xi_{l j}$ are independent if $i \neq j$, and each have expectation $1 / m$, and variance $(m-1) / m^{2}$. We will use the fact that the covariance of $\xi_{l i}$ and $\xi_{k i}$ is negative: it can be computed as $-1 / m^{2}$. 
For each $\pi \in \Pi$ we set $x_{l}(\pi)=\left(\sum_{j \in J_{l}} f_{j}^{2}\right)^{1 / 2}$. Then for $1 \leq r \leq 5^{m}$ we define $A(\pi, r)$ to be the set of $\omega \in \Omega$ such that $\left(\sum_{l=1}^{m}\left|b_{l}^{r}\right|^{2}\left|x_{l}(\pi, \omega)\right|^{2}\right)^{1 / 2} \leq$ $m^{-1 / 2}\left(F(\omega)+2.5^{m} F_{\infty}(\omega)\right)$.

Let us fix $\omega$ and $1 \leq r \leq 5^{m}$, and consider the random variable $\zeta$ on $\Pi$ defined by

$$
\zeta(\pi)=\sum_{l=1}^{m}\left|b_{l}^{r}\right|^{2} \sum_{j=1}^{n} \xi_{l j}(\pi)\left|f_{j}(\omega)\right|^{2}=\sum_{l=1}^{m}\left|b_{l}^{r}\right|^{2}\left|x_{l}(\pi, \omega)\right|^{2} .
$$

Then

$$
\int \zeta d P=\frac{1}{m} F(\omega)^{2} .
$$

Next we estimate the variance of $\zeta$ recalling our previous observations concerning the random variables $\xi_{k i}$.

$$
\int\left(\zeta-\frac{1}{m} F(\omega)^{2}\right)^{2} d P \leq \frac{1}{m} \sum_{l=1}^{m}\left|b_{l}^{r}\right|^{4} \sum_{j=1}^{n}\left|f_{j}(\omega)\right|^{4} \leq \frac{1}{m} F(\omega)^{2} F_{\infty}(\omega)^{2} .
$$

Now

$$
\int_{\omega \notin A(\pi, r)}\left(\zeta-\frac{1}{m} F(\omega)^{2}\right)^{2} d P \geq \frac{4.5^{m}}{m} F(\omega)^{2} F_{\infty}(\omega)^{2} P(\omega \notin A(\pi, r)) .
$$

Thus

$$
P(\omega \notin A(\pi, r)) \leq \frac{1}{4.5^{m}},
$$

Let $B(\pi, r)$ be the complement of $A(\pi, r)$. Then

$$
\frac{1}{m^{n}} \sum_{\pi \in \Pi} \int_{\Omega} \phi \chi_{B(\pi, r)} d \mu \leq \frac{1}{4.5^{m}} \int_{\Omega} \phi d \mu
$$

Summing over $r$ we further obtain the existence of some $\pi$ so that

$$
\int_{\Omega} \phi \sum_{r=1}^{5^{m}} \chi_{B(\pi, r)} d \mu \leq \frac{1}{4} \int_{\Omega} \phi d \mu .
$$

For this fixed $\pi$, let $B=\cup_{r=1}^{5^{m}} B(\pi, r)$. Then $\int_{B} \phi d \mu \leq \frac{1}{4} \int_{\Omega} \phi d \mu$. Let $A$ be the complement of $B$. Then $\int_{A} \phi d \mu \geq \frac{3}{4} \int_{\Omega} \phi d \mu$.

For $1 \leq r \leq 5^{m}$ we then have that

$$
\chi_{A}\left(\sum_{l=1}^{5^{m}}\left|b_{l}^{r}\right|^{2} x_{l}(\pi)^{2}\right)^{1 / 2} \leq m^{-1 / 2}\left(F+2.5^{m} F_{\infty}\right) .
$$

By considering the map $T: \ell_{2}^{m} \rightarrow X\left(\ell_{2}\right)$ defined by $T\left(e_{l}\right)=\left(0, \ldots, 0, x_{l}, 0, \ldots\right)$ with $x_{l}$ in the $l$ th. position it follows that for every $a_{1}, \ldots, a_{m}$ we have

$$
\left\|\left(\sum_{l=1}^{m}\left|a_{l}\right|^{2}\left|x_{l}\right|^{2}\right)^{1 / 2} \chi_{A}\right\|_{X} \leq 2 m^{-1 / 2}\left(\|F\|_{X}+2.5^{m}\left\|F_{\infty}\right\|_{X}\right)\left(\sum_{l=1}^{m}\left|a_{l}\right|^{2}\right)^{1 / 2} .
$$


Lemma 3.3. Let $\mathcal{X}$ be a lattice anti-Euclidean family of Banach sequence spaces. Then, given $M$ there exists $\delta=\delta(M)>0$ with the following property. Suppose that $Y$ is an order-continuous Köthe function space on $(\Omega, \mu)$ and $X \in \mathcal{X}$. Let $d=\operatorname{dim} X \leq \infty$. Suppose $S: X \rightarrow Y$ and $T: Y \rightarrow X$ are bounded operators with $\|S\|,\|T\| \leq M$. Suppose $\left(h_{n}\right)_{n=1}^{d} \in L_{1}(\mu)$ satisfy $0 \leq h_{n} \leq\left|S e_{n} \| T^{*} e_{n}\right|$ and $\int h_{n} d \mu \geq M^{-1}$. Then, for any $N \leq d$, and any $\alpha_{1}, \ldots, \alpha_{N}$,

$$
\int_{\Omega} \max _{1 \leq k \leq N}\left|\alpha_{k} h_{k}\right| d \mu \geq \delta \sum_{k=1}^{N}\left|\alpha_{k}\right|
$$

Proof. Let us suppose that for some $M$ the conclusion of the lemma is false. Suppose $m \in \mathbf{N}$ is given. We put $\epsilon=\left(2.5^{m}\right)^{-1}$. Then we can find $X \in \mathcal{X}$ and $S: X \rightarrow Y, T: Y \rightarrow X$ with $\|S\|,\|T\| \leq M$ and $0 \leq h_{n} \leq\left|S e_{n} \| T^{*} e_{n}\right|$ for $1 \leq n \leq d=\operatorname{dim} X$ with $\int h_{n} d \mu \geq M^{-1}$ and such that for suitable $0 \leq \zeta \in c_{00}$ and $N \in \mathbf{N}$, with $\|\zeta\|_{1}=1$ and $\zeta(i)=0$ for all $i>N$, we have

$$
\int_{\Omega} \max _{1 \leq k \leq N} \zeta(k) h_{k} d \mu<\frac{\epsilon^{2}}{16 M^{4}}
$$

Let $f_{n}=S e_{n}$ and $g_{n}=T^{*} e_{n}$. Notice that this implies that

$$
\left\|h_{n}\right\|_{1} \leq\left\|S e_{n}\right\|_{Y}\left\|T^{*} e_{n}\right\|_{Y^{*}} \leq M^{2}
$$

It follows from Krivine's theorem ([20] Theorem 1.f.4, p.93) that if $\alpha_{1}, \ldots, \alpha_{n} \in$ $\mathbf{R}$ then

$$
\begin{gathered}
\left\|\left(\sum_{k=1}^{n} \alpha_{k}^{2} f_{k}^{2}\right)^{1 / 2}\right\|_{Y} \leq K_{G} M\left\|\sum_{k=1}^{n} \alpha_{k} e_{k}\right\|_{X}, \\
\left\|\left(\sum_{k=1}^{n} \alpha_{k}^{2} g_{k}^{2}\right)^{1 / 2}\right\|_{Y^{*}} \leq K_{G} M\left\|\sum_{k=1}^{n} \alpha_{k} e_{k}\right\|_{X^{*}},
\end{gathered}
$$

where $K_{G}$ is as usual the Grothendieck constant.

By a well-known theorem of Lozanovskii we can factorize $\zeta=\xi \xi^{*}$ where $0 \leq$ $\xi, \xi^{*} \in c_{00}$ have the same support as $\zeta$ and satisfy $\|\xi\|_{X}=\left\|\xi^{*}\right\|_{X^{*}}=1$.

Next let $F=\left(\sum_{k=1}^{N} \xi(k)^{2} f_{k}^{2}\right)^{1 / 2} \in Y$. It follows from the remarks above that $\|F\|_{Y} \leq K_{G} M$. Similarly if $G=\left(\sum_{k=1}^{N} \xi^{*}(k)^{2} g_{k}^{2}\right)^{1 / 2} \in Y^{*}$ then $\|G\|_{Y^{*}} \leq K_{G} M$. Finally let $H=\sum_{k=1}^{n} \zeta(k) h_{k}$. Then $M^{-1} \leq\|H\|_{1}=\int H d \mu \leq M^{2}$.

For each $k$ let $B_{k}=\left\{\xi(k) f_{k} \geq \epsilon F\right\}$ and $B_{k}^{*}=\left\{\xi^{*}(k) g_{k} \geq \epsilon G\right\}$. We will let $A_{k}=\Omega \backslash\left(B_{k} \cup B_{k}^{*}\right)$. 
Now if $\omega \in B_{k}$ we have $F \xi^{*}(k) g_{k}(\omega) \leq \epsilon^{-1} \zeta(k) h_{k}(\omega)$ and so

$$
\begin{aligned}
\sum_{k=1}^{N} \zeta(k) h_{k} \chi_{B_{k}} & \leq\left(\sum_{k=1}^{N} \xi(k)^{2} f_{k}^{2} \chi_{B_{k}}\right)^{1 / 2}\left(\sum_{k=1}^{N} \xi^{*}(k)^{2} g_{k}^{2}\right)^{1 / 2} \\
& \leq F\left(\sum_{k=1}^{N} \xi^{*}(k)^{2} g_{k}^{2}\right)^{1 / 2} \\
& \leq \epsilon^{-1}\left(\sum_{k=1}^{N} \zeta(k)^{2} h_{k}^{2}\right)^{1 / 2} \\
& \leq \epsilon^{-1} H^{1 / 2}\left(\max _{1 \leq k \leq N} \zeta(k) h_{k}\right)^{1 / 2}
\end{aligned}
$$

From this we deduce that

$$
\left\|\sum_{k=1}^{N} \zeta(k) h_{k} \chi_{B_{k}}\right\|_{1} \leq \frac{1}{4 M^{2}}\|H\|_{1}^{1 / 2} \leq \frac{1}{4 M}
$$

Similarly

$$
\left\|\sum_{k=1}^{N} \zeta(k) h_{k} \chi_{B_{k}^{*}}\right\|_{1} \leq \frac{1}{4 M}
$$

Hence

$$
\int \sum_{k=1}^{N} \zeta(k) h_{k} \chi_{A_{k}} d \mu \geq \frac{1}{2 M}
$$

Now $\max _{1 \leq j \leq N} \xi(j) f_{j} \chi_{A_{j}} \leq \epsilon F$ while $\max _{1 \leq j \leq N} \xi^{*}(j) g_{j} \chi_{A_{j}} \leq \epsilon G$. Consider $X \oplus X^{*}$ (with the maximum norm) as a Köthe function space on two copies of $(\Omega, \mu)$ and consider the functions $\left(\xi(j) f_{j} \chi_{A_{j}}, \xi^{*}(j) g_{j} \chi_{A_{j}}\right)$ in $X \oplus X^{*}$. Using Lemma 3.2 with $\phi=\left(\sum_{k=1}^{N} \zeta(k) h_{k} \chi_{A_{k}}, \sum_{k=1}^{N} h_{k} \chi_{A_{k}}\right)$ it is easy to deduce the existence of a Borel subset $D$ of $\Omega$ with

$$
\int_{D} \sum_{k=1}^{N} \zeta(k) h_{k} \chi_{A_{k}} d \mu \geq \frac{1}{2 M}
$$

and a partition $J_{1}, \ldots, J_{m}$ of $[N]$ so that for any $a_{1}, \ldots, a_{m}$ we have

$$
\left\|\left(\sum_{k=1}^{m} a_{k}^{2} \sum_{j \in J_{k}} \xi(j)^{2} f_{j}^{2} \chi_{A_{j}}\right)^{1 / 2} \chi_{D}\right\|_{X} \leq 4 K_{G} M m^{-1 / 2}\left(\sum_{k=1}^{m} a_{k}^{2}\right)^{1 / 2}
$$

and

$$
\left\|\left(\sum_{k=1}^{m} a_{k}^{2} \sum_{j \in J_{k}} \xi^{*}(j)^{2} g_{j}^{2} \chi_{A_{j}}\right)^{1 / 2} \chi_{D}\right\|_{X^{*}} \leq 4 K_{G} M m^{-1 / 2}\left(\sum_{k=1}^{m} a_{k}^{2}\right)^{1 / 2}
$$


Let $L$ be the set of $1 \leq j \leq N$ so that $\left\|h_{j} \chi_{A_{j} \cap D}\right\|_{1} \geq \frac{1}{4 M}$. Then

$$
\int_{D} \sum_{j \notin L} \zeta(j) h_{j} \chi_{A_{j}} d \mu \leq \frac{1}{4 M}
$$

so that

$$
\int_{D} \sum_{j \in L} \zeta(j) h_{j} \chi_{A_{j}} d \mu \geq \frac{1}{4 M}
$$

Now let

$$
u_{k}=m^{1 / 2} \sum_{j \in J_{k} \cap L} \xi(j) e_{j} \in X
$$

and

$$
u_{k}^{*}=m^{1 / 2} \sum_{j \in J_{k} \cap L} \xi^{*}(j) e_{j} \in X^{*} .
$$

Consider an element $v=\sum_{k=1}^{m} a_{k} u_{k} \in X$ and let $v^{*}=\sum_{j \in L} v^{*}(j) e_{j} \in X^{*}$ norm $v$, i.e. $\left\|v^{*}\right\|_{X^{*}}=1$ and $\left\langle v, v^{*}\right\rangle=\|v\|_{X}$.

Then

$$
\begin{aligned}
\|v\|_{X} & \leq 4 M \sum_{j \in L} v(j) v^{*}(j) \int h_{j} \chi_{A_{j} \cap D} d \mu \\
& \leq 4 M \int\left(\sum_{j \in L} v(j)^{2} f_{j}^{2} \chi_{A_{j} \cap D}\right)^{1 / 2}\left(\sum_{j \in L} v^{*}(j)^{2} g_{j}^{2}\right)^{1 / 2} d \mu \\
& \leq 4 M\left\|\left(\sum_{j \in L} v(j)^{2} f_{j}^{2} \chi_{A_{j} \cap D}\right)^{1 / 2}\right\|_{X}\left\|\left(\sum_{j \in L} v^{*}(j)^{2} g_{j}^{2}\right)^{1 / 2}\right\|_{X^{*}} .
\end{aligned}
$$

Here the first factor can be estimated by $4 K_{G} M\left(\sum_{k=1}^{N} a_{k}^{2}\right)^{1 / 2}$ and the second factor by Krivine's theorem is majorized by $K_{G} M$. Hence

$$
\|v\|_{X} \leq 2^{4} K_{G}^{2} M^{3}\left(\sum_{k=1}^{m} a_{k}^{2}\right)^{1 / 2} .
$$

Thus we have the inequality'

$$
\left\|\sum_{k=1}^{m} a_{k} u_{k}\right\|_{X} \leq 2^{4} K_{G}^{2} M^{3}\left(\sum_{k=1}^{m} a_{k}^{2}\right)^{1 / 2} .
$$

Precisely dual arguments will yield that

$$
\left\|\sum_{k=1}^{m} a_{k} u_{k}^{*}\right\|_{X^{*}} \leq 2^{4} K_{G}^{2} M^{2}\left(\sum_{k=1}^{m} a_{k}^{2}\right)^{1 / 2} .
$$

Finally $\sum_{k=1}^{m}\left\langle u_{k}, u_{k}^{*}\right\rangle=m \sum_{j \in L} \zeta(j) \geq \frac{1}{4} m \int_{D} \sum_{j \in L} \zeta(j) h_{j} \chi_{A_{j}} \geq 2^{-4} M^{-1} m$.

Now Lemma 3.1 yields that $\ell_{2}^{m_{0}}$ is $2^{9} K_{G}^{4} M^{7}$-complementably $2^{9} K_{G}^{4} M^{7}$-lattice finitely representable in $X$ for some $m_{0} \geq 2^{-9} K_{G}^{-4} M^{-5} m$.

It is clear that this impossible for arbitrarily large $m$. 
Theorem 3.4. Let $Y$ be a nonatomic order-continuous Banach lattice and suppose that $\left(f_{n}\right)$ is a complemented unconditional basic sequence in $Y$. Suppose $\left(f_{n}\right)$ is lattice anti-Euclidean. Then $\left(f_{n}\right)$ is equivalent to a complemented disjoint sequence $\left(f_{n}^{\prime}\right)$ in $Y$.

Proof. We suppose that $Y$ is an order-continuous Köthe function space on $(\Omega, \mu)$, where $\mu$ is nonatomic. Let $X$ be the sequence space induced by $\left(f_{n}\right)$, and let $S: X \rightarrow Y$ be the bounded linear map with $S e_{n}=f_{n}$. Then there is also a bounded linear map $T: Y \rightarrow X$ with $T S=I_{X}$. As before let $g_{n}=T^{*} e_{n}$ and $h_{n}=\left|f_{n} g_{n}\right|$. Then for suitable $\delta>0$ we have

$$
\int_{\Omega} \max _{1 \leq k \leq N}\left|\alpha_{k} h_{k}\right| \geq \delta \sum_{k=1}^{N}\left|\alpha_{k}\right|
$$

for every $N, \alpha_{1}, \ldots, \alpha_{N}$. By a result of Dor [9] there exist disjoint Borel sets $\left(E_{n}\right)_{n=1}^{\infty}$ so that $\int_{E_{n}} h_{n} d \mu=\delta$. It is then easy to verify that $\left(\left|f_{n}\right| \chi_{E_{n}}\right)_{n=1}^{\infty}$ is a complemented disjoint sequence equivalent to $\left(f_{n}\right)$. Indeed define $U: X \rightarrow Y$ by $U e_{n}=f_{n} \chi_{E_{n}}$ and $V: Y \rightarrow X$ by $V(y)(j)=\delta^{-1}\left\langle y,\left|g_{j}\right| \chi_{E_{j}}\right\rangle$. Then $V U=I_{X}$ and for any $\xi \in c_{00}$ we have

$$
\|U \xi\|_{Y} \leq\left\|\left(\sum_{j=1}^{\infty} \xi(j)^{2} f_{j}^{2}\right)^{1 / 2}\right\|_{Y} \leq K_{G} M\|\xi\|_{X}
$$

Also if $\xi^{*} \in c_{00}$ then

$$
\left|\left\langle V y, \xi^{*}\right\rangle\right| \leq \delta^{-1}\left\langle|y|,\left(\sum_{j=1}^{\infty} \xi^{*}(j)^{2} g_{j}^{2}\right)^{1 / 2}\right\rangle \leq \delta^{-1} K_{G} M\left\|\xi^{*}\right\|_{X^{*}}
$$

Thus $U, V$ are both bounded operators and the theorem is proved.

Unfortunately if $Y$ is a sequence space the result is not quite so clean. We first state the corresponding theorem and then a more general technical result which includes the theorem.

Theorem 3.5. Let $Y$ be a Banach sequence space and suppose that $\left(f_{n}\right)$ is a complemented unconditional basic sequence in $Y$. Suppose $\left(f_{n}\right)$ is lattice antiEuclidean. Then $\left(f_{n}\right)$ is equivalent to a complemented disjoint sequence $\left(f_{n}^{\prime}\right)$ in $Y^{N}$ for some natural number $N$. 
Theorem 3.6. Let $\mathcal{X}$ be a lattice anti-Euclidean family of Banach sequence spaces. Then given $M>1$ there is a constant $C=C(M)$ and a natural number $N=N(M)$ so that the following property holds.

Suppose $Y$ is a Banach sequence space and $X \in \mathcal{X}$ with $\operatorname{dim} X=d \leq \infty$. Suppose $S: X \rightarrow Y$ and $T: Y \rightarrow X$ are bounded operators with $\|S\|,\|T\| \leq M$. Let $f_{n}=S e_{n}$ and $g_{n}=T^{*} e_{n}$ for $n \leq d$ and suppose $E_{n}$ are disjoint subsets of $\mathbf{N}$ so that $\left\|f_{n} g_{n} \chi_{E_{n}}\right\|_{1} \geq M^{-1}$. Then we can find subsets $\left(F_{k n}\right)$ of $\mathbf{N}$ for $1 \leq k \leq N$ and $1 \leq n \leq d$ so that (1) $F_{k n} \subset E_{n}$, (2) for each fixed $k$, the sets $\left(F_{k n}\right)$ are pairwise disjoint, (3) for each fixed $n$, the sets $\left(F_{k n}\right)$ are pairwise disjoint and (4) the disjoint sequence $\left(f_{n}^{\prime}\right)_{n=1}^{d}$ defined by $f_{n}^{\prime}=\left(f_{n} \chi_{F_{k n}}\right)_{k=1}^{N}$ in $Y^{N}$ is $C$-complemented and $C$-equivalent to the unit vectors $\left(e_{n}\right)_{n=1}^{d}$ in $X$.

Remark. Of course if we take $\mathcal{X}$ as having one member and $E_{n}=\mathbf{N}$, this implies Theorem 3.5. However, the quantitative version will be of some importance.

Proof. Let $\delta=\delta(M, \mathcal{X})$ be determined as in Lemma 3.3. We will show that $N=\left[2 M \delta^{-1}\right]$ and $C=2 K_{G}^{2} \delta^{-1} M^{2} N^{2}$ have the property claimed.

Let $h_{n}=\left|f_{n} g_{n}\right| \chi_{E_{n}}$. Then by Lemma 3.3 we can define an operator $R: \ell_{1} \rightarrow$ $\ell_{1}\left(c_{0}\right)$ by $R(\zeta)=\left(\zeta(k) h_{k}\right)_{k=1}^{\infty}$. (For notational convenience we will assume that $d=\operatorname{dim} X=\infty$; minor modifications can be made if $d<\infty$.) Now $\|R\| \leq M^{2}$ and $\|R(\zeta)\| \geq \delta\|\zeta\|_{1}$ for all $\zeta \in \ell_{1}$. We therefore can apply the Hahn-Banach theorem to find a linear functional $\Phi=\left(\phi_{n}\right)_{n=1}^{\infty} \in \ell_{\infty}\left(\ell_{1}\right)$ so that $\|\Phi\| \leq 1$ and $\Phi(R \zeta)=\delta \sum_{n=1}^{\infty} \zeta(n)$ for all $\zeta \in \ell_{1}$. In other words,

$$
\sup _{k} \sum_{n=1}^{\infty}\left|\phi_{n}(k)\right| \leq 1
$$

and

$$
\sum_{k=1}^{\infty} \phi_{n}(k) h_{n}(k)=\delta
$$

for each $n$.

Let $A_{n}=\left\{k:\left|\phi_{n}(k)\right| \geq 1 /(M N)\right\}$. Then

$$
\sum_{k \in A_{n}}\left|\phi_{n}(k)\right| h_{n}(k) \geq \frac{1}{2} \delta .
$$

Now $\sum_{n=1}^{\infty} \chi_{A_{n}}(k) \leq N \sum_{n=1}^{\infty}\left|\phi_{n}(k)\right| \leq N$. It follows that we can decompose $A_{n}=\cup_{k=1}^{N} F_{k n}$ as a disjoint union where for each $1 \leq k \leq N$ the sets $\left(F_{k n}\right)_{n=1}^{\infty}$ are disjoint. 
Let $f_{n}^{\prime}=\left(\left|f_{n}\right| \chi_{F_{k n}}\right)_{k=1}^{N} \in Y^{N}$ (which we consider as an $\ell_{2}$-sum). Similarly, let $g_{n}^{\prime}=\left(\left|g_{n}\right| \chi_{F_{k n}}\right)_{k=1}^{N} \in\left(Y^{*}\right)^{N}$. Then

$$
\left\langle f_{n}^{\prime}, g_{n}^{\prime}\right\rangle=\sum_{k \in A_{n}} h_{n}(k) \geq \sum_{k \in A_{n}}\left|\phi_{n}(k)\right| h_{n}(k) \geq \frac{1}{2} \delta .
$$

Let $\beta_{n}=\left\langle f_{n}^{\prime}, g_{n}^{\prime}\right\rangle$ and define $U: X \rightarrow Y^{N}$ and $V: Y^{N} \rightarrow X$ by $U(\xi)=$ $\sum_{j=1}^{\infty} \xi(j) f_{j}^{\prime}$ and $V(\mathbf{y})(j)=\beta_{j}^{-1}\left\langle\mathbf{y}, g_{j}^{\prime}\right\rangle$ where $\mathbf{y}=\left(y_{1}, \ldots, y_{N}\right)$.

Suppose $\xi \in c_{00}$. Then

$$
\|U \xi\|_{Y^{N}} \leq N\left\|\max _{j \geq 1}\left|\xi(j) f_{j}\right|\right\|_{Y} \leq N\left\|\left(\sum_{j=1}^{\infty} \xi(j)^{2} f_{j}^{2}\right)^{1 / 2}\right\|_{Y} \leq K_{G} M N\|\xi\|_{X} .
$$

From this it quickly follows that $U$ is well-defined and bounded with $\|U\| \leq$ $K_{G} M N$. On the other hand if $\xi \in c_{00}$ then

$$
\langle V \mathbf{y}, \xi\rangle \leq 2 \delta^{-1}\left\langle\sum_{k=1}^{N}\left|y_{k}\right|, \max _{j \geq 1}\left|\xi(j) g_{j}\right|\right\rangle \leq 2 K_{G} \delta^{-1} M N\|\mathbf{y}\|_{Y^{N}}
$$

so that $\|V\| \leq 2 K_{G} \delta^{-1} M N$. Since $V U=I_{X}$ the proof is complete.

Remarks. It is not possible to improve Theorem 3.5 by replacing $Y^{N}$ by $Y$. We sketch an example. Gowers [12] (cf [13]) has constructed a sequence space with the property that every bounded operator is a strictly singular perturbation of a diagonal operator. Let $1<p<2$ and consider the space $\tilde{G}=G\left(\ell_{p}^{2^{n}}\right)$ (i.e. the direct sum in the sense of $G$ of spaces $\ell_{p}^{2^{n}}$.) The obvious basis is antilattice Euclidean (in fact $\tilde{G}$ is $p$-concave). However $\tilde{G}$ has another unconditional basis which is formed by taking the Haar basis in each co-ordinate. It may be shown that the original basis is not equivalent to a block basis of this basis. We remark, however, that, in this example $N=2$ suffices and we know of no example where $N=2$ does not suffice. A somewhat similar problem is considered by Wojtaszczyk [26] for certain types of bases in quasi-Banach spaces.

We also remark that a continuous analogue of Theorems 3.4 and 3.5 is proved by somewhat similar techniques in [14], Theorem 8.1. This result which follows from work in [16] was, in fact, the basis for the proof given here. We have opted however for a completely self-contained approach. 


\section{Applications to Finite-Dimensional spaCes}

Before stating our first application, let us recall some definitions from [7]. Let $\mathcal{X}$ be a family of finite-dimensional Banach sequence spaces. Suppose first each $X \in \mathcal{X}$ is a symmetric space. Then we say the members of $\mathcal{X}$ have a unique symmetric basis if there is a function $\psi:[1, \infty) \rightarrow[1, \infty)$ so that if $\left(u_{i}\right)_{i=1}^{\operatorname{dim} X}$ is a normalized $K$-symmetric basis of some $X \in \mathcal{F}$ then $\left(u_{i}\right)_{i=1}^{\operatorname{dim} X}$ is $\psi(K)$-equivalent to $\left(e_{i}\right)_{i=1}^{\operatorname{dim} X}$.

Now consider the case when each $X$ is not necessarily symmetric.

Then we say the members of $\mathcal{X}$ have an almost (somewhat) unique unconditional basis if there is a function $\phi:[1, \infty) \times(0,1)$ so that given $K \geq 1$, then for any $0<\alpha<1$ (resp. for some $0<\alpha=\alpha(K)<1$ ) it is true that whenever $X \in \mathcal{X}$ has a normalized $K$-unconditional basis $\left(u_{i}\right)_{i=1}^{\operatorname{dim}} X$ then there is a subset $\sigma$ of $[\operatorname{dim} X]$ with $|\sigma| \geq \alpha \operatorname{dim} X$ and a one-one map $\pi: \sigma \rightarrow[\operatorname{dim} X]$ so that $\left(e_{i}\right)_{i \in \sigma}$ is $\phi(K, \alpha)$-equivalent to $\left(u_{\pi(i)}\right)_{i \in \sigma}$.

The following theorem shows that any collection of finite-dimensional spaces which form a lattice anti-Euclidean family (i.e. do not have uniformly complemented $\ell_{2}^{n}$-sublattices) have almost unique unconditional bases. In particular in any such class the symmetric basis is unique; both these results are new. There are, however, numerous results of this type in the literature. It was shown by Gowers [11] that the symmetric basis is not unique for the class of all symmetric spaces, but positive results for various classes are given in [4], [7], [15] and [24]. The problem of almost or somewhat uniqueness for unconditional bases for various classes was considered in [24] and [7].

Theorem 4.1. Let $\mathcal{X}$ be a lattice anti-Euclidean family of finite-dimensional sequence spaces. Then the members of $\mathcal{X}$ have almost unique unconditional bases.

In order to prove this we will need a lemma, due essentially to Wojtaszczyk [26]. Our statement is a modification and we will avoid the language of bipartite graph theory. Suppose $n \in \mathbf{N}$ and let $G$ be a subset of $[n] \times[n]$. For $i \in[n]$ let $V_{i}$ be the set of $j$ so that for some $k \in[n],(i, k)$ and $(j, k) \in G$. For $A \subset[n]$ let $V(A)=V^{1}(A)=\cup_{i \in A} V_{i}$ and then define inductively $V^{r}(A)=V\left(V^{r-1}(A)\right)$; let $V_{i}^{r}=V^{r}(\{i\})$. Finally let $G^{r}$ be the set of $(i, j)$ so that for some $k \in[n]$ we have $(k, j) \in G$ and $k \in V_{i}^{r}$.

Lemma 4.2. Assume $G$ is as above. Suppose $\left(w_{i j}\right)_{i, j \in[n]}$ are such that: (1) $\sum_{j=1}^{n} w_{i j}=1$ for $i \in[n]$, 
(2) $\sum_{i=1}^{n} w_{i j}=1$ for $j \in[n]$.

Let $M=\max _{1 \leq j \leq n} \sum_{i=1}^{n}\left|w_{i j}\right|$ and let $b=\sum_{(i, j) \notin G}\left|w_{i j}\right|$. Then for any $r$ there is a subset $\sigma$ of $[n]$ with $|\sigma| \geq n-3 b-M r^{-1} n$ and a one-one map $\pi: \sigma \rightarrow[n]$ with $(i, \pi(i)) \in G^{r}$ for $i \in \sigma$.

Proof. Note first that either $V_{i}$ is empty or $i \in V_{i}$; let $E$ be the set of $i$ such that $V_{i}$ is empty. Then $|E|=\sum_{i \in E} \sum_{j=1}^{n} w_{i j} \leq b$. For any $A$ we have $A \subset V(A) \cup E$ and $V(A) \cap E=\emptyset$. Thus the sequence $\left(V^{s}(A)\right)_{s=1}^{n}$ is increasing and so for every $1 \leq s \leq r$ we have

$$
\left|V^{s}(A)\right| \geq|A|-b
$$

For future reference we let $V^{0}(A)=A \backslash E$ and have the same inequality. Now for any $A \subset[n]$ let $A^{*}=\{j: \exists i \in A,(i, j) \in G\}$ and $A^{+}=\left\{j: \exists i \in A:(i, j) \in G^{r}\right\}$. Then $A^{+}=\left(V^{r}(A)\right)^{*}$.

Assume for some $A$ we have $\left|A^{+}\right| \leq|A|$. Then there exists some $0 \leq s \leq r-1$ so that $\left|V^{s+1}(A)^{*}\right| \leq\left|V^{s}(A)^{*}\right|+r^{-1}|A|$. (Here we recall $V^{0}(A)=A \backslash E$.). Notice $V^{s+1}(A) \supset V^{s}(A)$. We now compute

$$
\begin{aligned}
\left|V^{s+1}(A)\right| & =\sum_{i \in V^{s+1}(A)} \sum_{j=1}^{n} w_{i j} \\
& \leq b+\sum_{i \in V^{s+1}(A)} \sum_{j \in V^{s+1}(A)^{*}} w_{i j} \\
& \leq b+M\left(\left|V^{s+1}(A)^{*}\right|-\left|V^{s}(A)^{*}\right|\right)+\sum_{i \in V^{s+1}(A)} \sum_{j \in V^{s}(A)^{*}} w_{i j} \\
& \leq 2 b+M r^{-1}|A|+\sum_{i=1}^{n} \sum_{j \in V^{s}(A)^{*}} w_{i j} \\
& =2 b+M r^{-1}|A|+\left|V^{s}(A)^{*}\right| .
\end{aligned}
$$

Hence

$$
|A| \leq 3 b+M r^{-1}|A|+\left|A^{+}\right|
$$

Combining we have $\left|A^{+}\right| \geq|A|-3 b-M r^{-1} n$. The result then follows from Hall's Marriage Lemma [2].

Proof of Theorem 4.1. It will suffice to show that for any $\epsilon>0$ and $M \geq 1$ there is a constant $C=C(\epsilon, M, \mathcal{X})$ with the property that whenever $X, Y$ are finitedimensional sequence spaces with $X \in \mathcal{X}$ and if $S: X \rightarrow Y$ is an isomorphism with 
$\max \left(\|S\|,\left\|S^{-1}\right\|\right) \leq M$ then there is a subset $\sigma$ of $[\operatorname{dim} X]$ with $|\sigma| \geq(1-\epsilon) \operatorname{dim} X$ and a one-one map $\pi: \sigma \rightarrow[\operatorname{dim} X]$ so that for any $\left(\alpha_{i}\right)_{i \in \sigma}$ we have

$$
C^{-1}\left\|\sum_{i \in \sigma} \alpha_{i} e_{i}\right\|_{X} \leq\left\|\sum_{i \in \sigma} \alpha_{i} e_{\pi(i)}\right\|_{Y} \leq C\left\|\sum_{i \in \sigma} \alpha_{i} e_{i}\right\|_{X}
$$

Let $n=\operatorname{dim} X$ and let $T=S^{-1}$. As before, let $S e_{i}=f_{i}$ and $T^{*} e_{i}=g_{i}$ for $1 \leq i \leq n$. Let $w_{i j}=f_{i}(j) g_{i}(j)$. Then $\sum_{i=1}^{n} w_{i j}=1$ for all $j$ and $\sum_{j=1}^{n} w_{i j}=1$ for all $i$. Furthermore, $\sum_{i=1}^{n}\left|w_{i j}\right|=\left\|\left|S^{*} e_{j}\left\|T e_{j} \mid\right\|_{1} \leq\left\|S^{*} e_{j}\right\|_{X^{*}}\left\|T e_{j}\right\|_{X} \leq M^{2}\right.\right.$.

Now by Lemma 3.3 there exists a $\delta=\delta(\epsilon, M)$ so that if for some subset $\tau$ of $[n]$ we choose $0 \leq h_{k} \leq\left|f_{k}\right|\left|g_{k}\right|$ for $k \in \tau$ so that $\left\|h_{k}\right\| \geq \epsilon / 8$ then

$$
\left\|\max _{k \in \tau}\left|\alpha_{k} h_{k}\right|\right\|_{1} \geq \delta \sum_{k \in \tau}\left|\alpha_{k}\right|
$$

for all $\left(\alpha_{k}\right)_{k \in \tau}$. Next choose $\eta=\frac{1}{8} \delta \epsilon M^{-2}$.

We let $G$ be the set of pairs $(i, j)$ so that $\left|w_{i j}\right| \geq \eta$. Then

$$
\sum_{(i, j) \notin G}\left|w_{i j}\right|=\sum_{i=1}^{n}\left\|f_{i} g_{i} \chi_{A_{i}}\right\|_{1}
$$

where $A_{i}=\left\{j:\left|w_{i j}\right|<\eta\right.$. Let $h_{i}=\left|f_{i} g_{i}\right| \chi_{A_{i}}$ and let $\tau=\left\{i:\left\|h_{i}\right\|_{1} \geq \frac{1}{8} \epsilon\right\}$. Then

$$
\delta|\tau| \leq\left\|\max _{i \in \tau} h_{i}\right\|_{1} \leq n \eta
$$

so that $|\tau| \leq \frac{1}{8} \epsilon M^{-2} n$. Hence $\sum_{i \in \tau}\left\|h_{i}\right\|_{1} \leq \frac{1}{8} \epsilon n$. However $\sum_{i \notin \tau}\left\|h_{i}\right\|_{1} \leq \frac{1}{8} \epsilon n$. Combining we have

$$
\sum_{(i, j) \notin G}\left|w_{i j}\right| \leq \frac{1}{4} \epsilon n .
$$

We can now apply Lemma 4.2. We choose $r=\left[4 M^{2} \epsilon\right]+1$ so that we have a subset $\sigma$ of $[n]$ with $|\sigma| \geq(1-\epsilon) n$ and a one-one map $\pi: \sigma \rightarrow[n]$ so that $(i, \pi(i)) \in G^{r}$ for $i \in \sigma$.

Note that if $(i, j) \in G$ then $\left|f_{i}(j)\right|,\left|g_{i}(j)\right| \geq \delta / M$. Now by Krivine's theorem if $\left(\alpha_{i}\right)_{i=1}^{n}$ are scalars then

$$
\left\|\left(\sum_{i=1}^{n} \alpha_{i}^{2} f_{i}^{2}\right)^{1 / 2}\right\|_{Y} \leq K_{G} M\left\|\sum_{i=1}^{n} \alpha_{i} e_{i}\right\|_{X}
$$

Hence

$$
\left\|\left(\sum_{(i, j) \in G} \alpha_{i}^{2} e_{j}\right)^{1 / 2}\right\|_{Y} \leq K_{G} M^{2} \delta^{-1}\left\|\sum_{i=1}^{n} \alpha_{i} e_{i}\right\|_{X}
$$


Similarly, a dual argument gives that

$$
\left\|\left(\sum_{(i, j) \in G} \alpha_{j}^{2} e_{i}\right)^{1 / 2}\right\|_{X} \leq K_{G} M^{2} \delta^{-1}\left\|\sum_{j=1}^{n} \alpha_{j} e_{j}\right\|_{Y} .
$$

Iterating these conditions gives that if $C=\left(K_{G}^{2} M^{2} \delta^{-1}\right)^{r+1}$ then

$$
\left\|\left(\sum_{(i, j) \in G^{r}} \alpha_{i}^{2} e_{j}\right)^{1 / 2}\right\|_{Y} \leq C\left\|\sum_{i=1}^{n} \alpha_{i} e_{i}\right\|_{X} .
$$

It follows that

$$
\left\|\sum_{i \in \sigma} \alpha_{i} e_{\pi(i)}\right\|_{Y} \leq C\left\|\sum_{i \in \sigma} \alpha_{i} e_{i}\right\|_{X}
$$

Similarly we have

$$
\left\|\left(\sum_{(i, j) \in G^{r}} \alpha_{j}^{2} e_{i}\right)^{1 / 2}\right\|_{Y} \leq C\left\|\sum_{j=1}^{n} \alpha_{j} e_{j}\right\|_{X},
$$

so that

$$
\left\|\sum_{i \in \sigma} \alpha_{i} e_{i}\right\|_{X} \leq C\left\|\sum_{i \in \sigma} \alpha_{i} e_{\pi(i)}\right\|_{Y}
$$

so that the result follows.

\section{Right- AND LEFT-DOMinANT SPACES}

Let $X$ be a sequence space. We will say that $X$ is left-dominant with constant $\gamma \geq 1$ if whenever $\left(u_{1}, u_{2}, \ldots, u_{n}\right)$ and $\left(v_{1}, \ldots, v_{n}\right)$ are two disjoint sequences in $c_{00}$ with $\left\|u_{k}\right\|_{X} \geq\left\|v_{k}\right\|_{X}$ and such that supp $v_{k}>\operatorname{supp} u_{k}$ for $1 \leq k \leq n$ then $\left\|\sum_{k=1}^{n} v_{k}\right\|_{X} \leq \gamma\left\|\sum_{k=1}^{n} u_{k}\right\|_{X}$. Similarly, we will say that $X$ is right-dominant with constant $\gamma$ if whenever $\left(u_{1}, \ldots, u_{n}\right)$ and $\left(v_{1}, \ldots, v_{n}\right)$ are two disjoint sequences with $\left\|u_{k}\right\|_{X} \leq\left\|v_{k}\right\|_{X}$ and supp $v_{k}>\operatorname{supp} u_{k}$ for $1 \leq k \leq n$ then $\left\|\sum_{k=1}^{n} u_{k}\right\|_{X} \leq \gamma\left\|\sum_{k=1}^{n} v_{k}\right\|_{X}$.

We will refer to any normalized unconditional basic sequence as being leftor right-dominant according as the associated sequence space is left- or rightdominant. 
Lemma 5.1. $X$ is left-dominant with constant $\gamma$ if and only if $X^{*}$ is rightdominant with constant $\gamma$.

Proof. Let us prove that if $X$ is left-dominant then $X^{*}$ is right-dominant; the other direction is similar. Suppose $\left(u_{1}^{*}, \ldots, u_{n}^{*}\right)$ and $\left(v_{1}^{*}, \ldots, v_{n}^{*}\right)$ are two sequences in $c_{00}$ with $\left\|u_{k}^{*}\right\|_{X^{*}} \leq\left\|v_{k}^{*}\right\|_{X^{*}}$ for $1 \leq k \leq n$ and $\operatorname{supp} u_{k}^{*}<\operatorname{supp} v_{k}^{*}$. There exists $x \in X$ supported on $\cup_{i=1}^{n} \operatorname{supp} u_{i}^{*}$ with $\|x\|=1$ and $\left\langle x, \sum_{i=1}^{n} u_{i}^{*}\right\rangle=$ $\left\|\sum_{i=1}^{n} u_{i}^{*}\right\|_{X^{*}}$. Let $x=\sum_{i=1}^{n} u_{i}$ where supp $u_{i} \subset \operatorname{supp} u_{i}^{*}$. Next pick $v_{i}$ of norm one with support contained in $\operatorname{supp} v_{i}^{*}$ so that $\left\langle v_{i}, v_{i}^{*}\right\rangle=\left\|v_{i}^{*}\right\|_{X^{*}}$. Finally let $y=\sum_{i=1}^{n}\left\|u_{i}\right\|_{X} v_{i}$. Then $\|y\| \leq \gamma\|x\|=\gamma$. Also

$$
\begin{aligned}
\left\langle y, \sum_{i=1}^{n} v_{i}^{*}\right\rangle & =\sum_{i=1}^{n}\left\|u_{i}\right\|_{X}\left\|v_{i}^{*}\right\|_{X^{*}} \\
& \geq \sum_{i=1}^{n}\left\langle u_{i}, u_{i}^{*}\right\rangle \\
& \geq\left\|\sum_{i=1}^{n} u_{i}^{*}\right\|_{X^{*}} . \square
\end{aligned}
$$

If $N$ is a natural number we denote by $X_{N}$ the space $X[N+1, \infty)$ of all $x \in X$ such that $x(k)=0$ when $k \leq N$.

Lemma 5.2. Suppose $X$ is a left-(resp. right-)dominant sequence space. Suppose $1 \leq p \leq \infty$ and $\ell_{p}$ is disjointly finitely representable in $X$. Then :

(1) X satisfies a lower-(resp. upper-) p-estimate.

(2) There is a constant $K$ so that for any $n \in \mathbf{N}$, there exists $N \in \mathbf{N}$ so that $X_{N}$ satisfies an upper- (resp. lower-) estimate with constant $K$ on $n$ vectors.

Proof. We consider only the case of a left-dominant space, and assume that $\ell_{p}$ is $C$-disjointly representable in $X$ (actually by Krivine's theorem [19] we could suppose $C=1$ ). For notational convenience suppose $p<\infty$. Suppose $x_{1}, \ldots, x_{n}$ are disjoint in $c_{00}$. Then there exist $y_{1}, \ldots, y_{n}$ disjoint in $X$ with $\max _{k} \operatorname{supp} x_{k}<\operatorname{supp} y_{j}$ for $1 \leq j \leq n$ such that $\left\|y_{j}\right\|=\left\|x_{j}\right\|$ and $\left\|\sum_{j=1}^{n} y_{j}\right\| \geq$ $(2 C)^{-1}\left(\sum_{j=1}^{n}\left\|x_{j}\right\|^{p}\right)^{1 / p}$. Thus $\left\|\sum_{j=1}^{n} x_{j}\right\| \geq(2 C \gamma)^{-1}\left(\sum_{j=1}^{n}\left\|x_{j}\right\|^{p}\right)^{1 / p}$. Conversely, if we fix $n$ and choose any $y_{1}, \ldots, y_{n}$ normalized, disjoint and $2 C$ equivalent to an $\ell_{p}^{n}$-basis then if $N=\max _{k} \operatorname{supp} y_{k}$ and $x_{1}, \ldots, x_{n}$ are disjoint in $X_{N}$ then $\left.\left\|\sum_{j=1}^{n} x_{j}\right\| \leq \gamma\left\|\sum_{j=1}^{n}\right\| x_{j}\left\|y_{j}\right\| \leq(2 C \gamma)\left\|\sum_{j=1}^{n}\right\| x_{j} \|^{p}\right)^{1 / p}$.

It follows from Lemma 5.2 and Krivine's theorem that if $X$ is left- or rightdominant then there is exactly one $r=r(X)$ so that $\ell_{r}$ is disjointly finitely 
representable in $X$. Let us call $r$ the index of $X$. If $X$ is right-dominant and of index $\infty$ then clearly $X=c_{0}$ while if $X$ is left-dominant of index 1 then $X=\ell_{1}$. A right-dominant space of finite index has a nontrivial lower estimate and so can realized as the dual of left-dominant space of index greater than one.

Notice that it also follows from Lemma 5.2 that every left- or right-dominant sequence space is an asymptotically $\ell_{r}$ space where $r=r(X)$ (cf. [23], p. 221). We now turn our attention to the problem of deciding when a left- or right-dominant space is sufficiently Euclidean.

Proposition 5.3. Let $X$ be a left- or right-dominant sequence space. Then $X$ is sufficiently Euclidean if and only if $1<r(X)<\infty$.

Proof. Let $\mathcal{U}$ be a nonprincipal ultrafilter on the natural numbers and let $X_{n}=$ $X[n, \infty)$. Let $Y$ be the ultraproduct $\ell_{\infty}\left(X_{n}\right) / c_{0, \mathcal{U}}\left(X_{n}\right)$ where $c_{0, \mathcal{U}}\left(X_{n}\right)$ consists of all sequences $\left(x_{n}\right) \in \ell_{\infty}\left(X_{n}\right)$ with $\lim _{n \in \mathcal{U}}\left\|x_{n}\right\|=0$. Then $X$ is sufficiently Euclidean if and only if $\ell_{2}$ embeds complementably into $Y$. Assume $X$ is leftor right-dominant with index $r$. Then $Y$ is a Banach lattice with an upper and lower $r$-estimate. This implies $Y$ is isomorphic to an abstract $L_{r}$-space and so the result follows.

Proposition 5.4. Suppose $\left(u_{n}\right)$ is a left- (resp. right-) dominant basis and that $\pi$ is a permutation of the natural numbers such that $\left(u_{\pi(n)}\right)$ is also left- (resp. right-)dominant. Then there is a constant $C$ such that for any $\alpha \in c_{00}$

$$
\left\|\sum_{k=1}^{\infty} \alpha_{k} u_{2 k}\right\| \leq C\left\|\sum_{k=1}^{\infty} \alpha_{k} u_{\pi(k)}\right\|
$$

(respectively,

$$
\left.\left\|\sum_{k=1}^{\infty} \alpha_{k} u_{2 k}\right\| \geq C^{-1}\left\|\sum_{k=1}^{\infty} \alpha_{k} u_{\pi(k)}\right\| .\right)
$$

Proof. We treat only the left-dominant case. Define a sequence $\left(s_{n}\right)$ inductively as follows. Let $s_{1}=1$ and then let $s_{n}$ be the least $m$ so that $m \in \pi\{n, n+$ $1, \ldots\} \backslash\left\{s_{1}, \ldots, s_{n-1}\right\}$. Note that $s_{n} \leq 2 n-1<2 n$ and that $\left(s_{n}\right)$ increases. Further $s_{n}=\pi\left(r_{n}\right)$ where $r_{n} \geq n$. Hence $\left(u_{\pi(n)}\right)$ dominates $\left(u_{s_{n}}\right)$ which in turn dominates $\left(u_{2 n}\right)$. This establishes the result.

Proposition 5.5. Let $\left(u_{n}\right)$ be a left- (resp. right-)dominant basis of a Banach space $X$. In order that $\left(u_{n}\right)$ be equivalent to its square it is necessary and sufficient that $\left(u_{n}\right)$ be equivalent to $\left(u_{2 n}\right)$. 
Remark. Clearly $\left(u_{n}\right)$ is equivalent to $\left(u_{2 n}\right)$ if and only if $\left(u_{n}\right)$ is equivalent to $\left(u_{N n}\right)$ for any $N$ in view of the dominance assumption.

Proof. Consider the left-dominant case and assume $\left(u_{n}\right)$ is equivalent to its square. Let $\left(v_{n}\right)$ be the natural basis of $X^{4}$ with $\left(v_{4 n-j}\right)$ equivalent to $\left(u_{n}\right)$, for $0 \leq j \leq 3$. Then $\left(v_{n}\right)$ is left-dominant. Since some permutation of $\left(v_{n}\right)$ is equivalent to $\left(u_{n}\right)$ we have that $\left(u_{n}\right)$ dominates $\left(v_{2 n}\right)$ and hence $\left(u_{2 n}\right)$ dominates $\left(u_{n}\right)$. This implies $\left(u_{n}\right)$ and $\left(u_{2 n}\right)$ are equivalent. The other case is similar and the other direction is trivial.

Theorem 5.6. Let $X$ be a left- or right-dominant separable sequence space and that $\left(u_{n}\right)$ is a complemented normalized disjoint sequence in $X$. Then $\left(u_{n}\right)$ is permutatively equivalent to a subsequence of the canonical unconditional basis $\left(e_{n}\right)$ of $X$.

Proof. Let us assume the basis is left-dominant; the case of a right-dominant basis is almost identical. We can assume the dual functionals $\left(u_{n}^{*}\right)$ in $X^{*}$ have the same support as $\left(u_{n}\right)$. Let $f_{n}=\left|u_{n}\right|\left|u_{n}^{*}\right| \in \ell_{1}$. For each $n$ pick $k_{n} \in \operatorname{supp} u_{n}$ so that $\left\|f_{n} e_{\left[1, k_{n}\right]}\right\|_{1} \geq \frac{1}{2}$ and $\left\|f_{n} e_{\left[k_{n}, \infty\right)}\right\|_{1} \geq \frac{1}{2}$. Let $v_{n}=u_{n} e_{\left[1, k_{n}\right]}$ and $w_{n}=u_{n} e_{\left[k_{n}, \infty\right)}$. Now we argue that $\left(v_{n}\right)$ and $\left(w_{n}\right)$ are both equivalent to $\left(u_{n}\right)$. Indeed the operator $T x=\sum_{n=1}^{\infty}\left\langle x,\left|u_{n}^{*}\right|\right\rangle u_{n}$ is easily seen to be bounded on $X$. We have $T\left|v_{n}\right|=\alpha_{n} u_{n}$, and $T\left|w_{n}\right|=\beta_{n} u_{n}$ where $\alpha_{n}, \beta_{n} \geq 1 / 2$. It follows that both $\left(\left|v_{n}\right|\right)$ and $\left(\left|w_{n}\right|\right)$ are equivalent to $\left(u_{n}\right)$ and the desired conclusion follows.

Now if $X$ is left-dominant $\left(v_{n}\right)$ dominates $\left(e_{k_{n}}\right)$; to see this just note that $\left(v_{n} e_{\left[1, k_{n}\right)}\right)$ dominates $\left(\left\|v_{n} e_{\left[1, k_{n}\right)}\right\| e_{k_{n}}\right)$. Similarly, $\left(e_{k_{n}}\right)$ dominates $\left(w_{n}\right)$. Thus $\left(u_{n}\right)$ is equivalent to $\left(e_{k_{n}}\right)$.

Theorem 5.7. Let $X$ be a separable left- or right- dominant sequence space. Suppose that $r(X)=1$ or $r(X)=\infty$ and that $\left(e_{n}\right)$ and $\left(e_{2 n}\right)$ are equivalent. Then every complemented normalized unconditional basic sequence is equivalent to a subsequence of the basis and $X$ has a unique unconditional basis.

Proof. We assume $X$ left-dominant. Let $\left(u_{n}\right)$ be any complemented normalized unconditional basic sequence in $X$. Then $\left(u_{n}\right)$ is anti-lattice Euclidean by Proposition 5.3 and so by Theorem 3.5 and the hypothesis on $X,\left(u_{n}\right)$ is equivalent to a complemented disjoint sequence in $X$. By Theorem 5.6, this implies that $\left(u_{n}\right)$ is permutatively equivalent to a subsequence $\left(e_{k_{n}}\right)$ of $\left(e_{n}\right)$.

We now restrict to the case when $\left(u_{n}\right)$ is an unconditional basis of $X$. Applying Theorem 3.5 again we see that $\left(e_{n}\right)$ is equivalent to a complemented disjoint 
sequence in the $N$-fold sum basis $\left(u_{n}\right)^{N}$ of $X^{N}$. Now $\left(e_{k_{n}}\right)^{N}$ arranged in the obvious order is also a left-dominant basis. Here the "obvious order" $\left(f_{n}\right)$ is to take $f_{N(j-1)+s}$ to be $\left(0, \ldots, 0, e_{k_{j}}, 0, \ldots\right) \in X^{N}$, where $e_{k_{j}}$ is in the $s$ th. coordinate. Hence $\left(e_{n}\right)$ is permutatively equivalent to a subset of $\left(e_{k_{n}}\right)^{N}$. However $\left(e_{k_{n}}\right)^{N}$ is permutatively equivalent to a subset of $\left(e_{n}\right)^{N}$ which is permutatively equivalent to $\left(e_{n}\right)$. By the Cantor-Bernstein principle [27], this means that $\left(e_{k_{n}}\right)^{N}$ is permutatively equivalent to $\left(e_{n}\right)$.

Now $\left(f_{k}\right)$ dominates $\left(e_{2 k}\right)$ by Proposition 5.4 and similarly $\left(e_{k}\right)$ dominates $\left(f_{2 k}\right)$. Hence, since $\left(e_{k}\right)$ and $\left(e_{4 k}\right)$ are equivalent we have that $\left(f_{2 k}\right)$ is equivalent to $\left(e_{k}\right)$. Now $\left(f_{2 n-1}\right)_{n \geq 1}$ is dominated by $\left(f_{1}, f_{2}, f_{4}, \ldots\right)$ and dominates $\left(f_{1}, f_{4}, f_{8}, \ldots\right)$ and thus is also equivalent to $\left(f_{2 n}\right)$. Hence $\left(f_{n}\right)$ is equivalent to $\left(e_{n}\right)$. Now $f_{N n}$ is equivalent to $e_{N n}$ and hence to $\left(e_{n}\right)$. Thus $\left(e_{k_{n}}\right)$ is equivalent to $\left(e_{n}\right)$. The result now follows.

Remarks. There is a natural question here, which is also suggested by the work of Wojtaszczyk [26]. Suppose $\left(x_{n}\right)$ and $\left(y_{n}\right)$ are two unconditional bases whose squares are permutatively equivalent; does it follow that $\left(x_{n}\right)$ and $\left(y_{n}\right)$ are permutatively equivalent? The corresponding Banach space problem has a negative solution. An example of Gowers [13] shows that there is a Banach space $X$ so that $X$ and $X^{2}$ are not isomorphic but $X^{2}$ and $X^{4}$ are isomorphic.

Theorem 5.8. Suppose that $1<p_{n}<\infty$ for all $n$ and that $p_{n} \downarrow 1$. Suppose that for some constant $a>0$

$$
\frac{1}{p_{2 n}}-\frac{1}{p_{n}} \leq \frac{a}{\log n}
$$

for $n \geq 2$. Then the Nakano space $\ell\left(p_{n}\right)$ has a unique unconditional basis.

Similarly if $p_{n} \uparrow \infty$ and for some constant $a>0$

$$
\frac{1}{p_{n}}-\frac{1}{p_{2 n}} \leq \frac{a}{\log n}
$$

for $n \geq 2$, then $h\left(p_{n}\right)$ has a unique unconditional basis.

Proof. If $p_{n} \downarrow 1$ then $X=\ell\left(p_{n}\right)$ has a right-dominant basis with $r(X)=1$. The assumption that $\frac{1}{p_{n}}-\frac{1}{p_{2 n}}=O\left((\log n)^{-1}\right)$ implies that the basic sequences $\left(e_{n}\right)$ and $\left(e_{2 n}\right)$ are equivalent by an old result of Simons [25]. The second case is similar (or dual). 
Theorem 5.9. Let $p_{n} \downarrow 1$ be such that $p_{n}=1+O(\log n)^{-1}$. Assume $\left(N_{n}\right)$ is an increasing sequence of natural numbers such that $\frac{1}{p_{n+1}}-\frac{1}{p_{n}}=O\left(\left(\log N_{n}\right)^{-1}\right)$ and $\inf _{n} N_{n+1} / N_{n}>1$. Then $\ell_{1}\left(\ell_{p_{n}}^{N_{n}}\right)$ has a unique unconditional basis.

Proof. Let $q_{n}$ be the sequence obtained by writing out $p_{1}, N_{1}$ times, $p_{2} N_{2}$ times etc. It is clear that the Nakano space $\ell\left(q_{n}\right)$ can be written as vectorvalued Nakano space $\ell\left(p_{n}\right)\left(\ell_{p_{n}}^{N_{n}}\right)$. But by Simons's theorem [25] $\ell\left(p_{n}\right)=\ell_{1}$. Let $M_{n}=N_{1}+\cdots+N_{n}$. Then $M_{n} \leq c N_{n}$ for some $c$. If $M_{n-1}<k \leq M_{n}$ and $M_{m-1}<2 k \leq M_{m}$ we have that either $m-n \leq 1$ or $(m-n-1) N_{n} \leq k$. Thus $(m-n)$ is bounded independent of $k$ and so $\frac{1}{q_{2 k}}-\frac{1}{q_{k}}=O\left(\left(\log N_{n}\right)^{-1}\right)=$ $O(\log k)^{-1}$. The result follows from Theorem 5.8.

Remark. We do not know if Theorem 5.9 holds for any space $\ell_{1}\left(\ell_{p_{n}}^{N_{n}}\right)$ where $p_{n} \downarrow 1$.

Our final example of this section is the now classical Tsirelson space. We refer to [8] for full details of this space. We recall that the Tsirelson norm \|\|$_{T}$ on $c_{00}$ is the minimal norm satisfying $\|x\|_{T} \geq\|x\|_{\infty}$ and

$$
\frac{1}{2} \sum_{j=1}^{n}\left\|x_{j}\right\|_{T} \leq\left\|\sum_{j=1}^{n} x_{j}\right\|_{T}
$$

whenever $n \leq \operatorname{supp} x_{1}<\operatorname{supp} x_{2}<\cdots<\operatorname{supp} x_{n}$. Tsirelson space is the sequence space $T$ obtained by completing $c_{00}$ with respect to this norm. This space is the dual of the original Tsirelson space. We will need an alternative norm \|\|$_{T}^{\#}$ which is defined to be the least norm satisfying $\|x\|_{T}^{\#} \geq\|x\|_{\infty}$ and

$$
\frac{1}{2} \sum_{j=1}^{2 n}\left\|x_{j}\right\|_{T}^{\#} \leq\left\|\sum_{j=1}^{2 n} x_{j}\right\|_{T}^{\#}
$$

whenever $x_{1}, \ldots, x_{2 n}$ are disjoint and $n \leq \operatorname{supp} x_{j}$ for $j=1,2, \ldots, 2 n$.

Lemma 5.10. For $x \in c_{00}$ we have $\|x\|_{T} \leq\|x\|_{T}^{\#} \leq 4\|x\|_{T}$.

Proof. Let \|\|$_{T}^{\prime}$ be the least norm on $c_{00}$ so that $\|x\|_{T}^{\prime} \geq\|x\|_{\infty}$ and

$$
\frac{1}{2} \sum_{j=1}^{2 n}\left\|x_{j}\right\|_{T}^{\prime} \leq\left\|\sum_{j=1}^{2 n} x_{j}\right\|_{T}^{\prime}
$$

whenever $n \leq \operatorname{supp} x_{1}<\operatorname{supp} x_{2}<\cdots<\operatorname{supp} x_{2 n}$. By [5] we have $\|x\|_{T} \leq$ $\|x\|_{T}^{\prime} \leq 2\|x\|_{T}$ and by [1] we have $\|x\|_{T}^{\prime} \leq\|x\|_{T}^{\#} \leq 2\|x\|_{T}^{\prime}$.

We now prove that Tsirelson space is right-dominant. This result was stated without proof in [3], and generalizes Lemma II.1 of [8]. 
Lemma 5.11. Suppose $x_{1}, \ldots, x_{N}$ are disjoint in $c_{00}$ and let $a_{k}=\max \operatorname{supp} x_{k}$.

Then

$$
\left\|\sum_{k=1}^{N} x_{k}\right\|_{T} \leq 4\left\|\sum_{k=1}^{N}\right\| x_{k}\left\|_{T} e_{a_{k}}\right\|_{T}^{\#} .
$$

Proof. Indeed if this inequality is false there exist disjoint $x_{1}, \ldots, x_{N}$ with support supp $\left(x_{1}+\cdots+x_{N}\right)$ of minimal cardinality such that

$$
\left\|\sum_{k=1}^{N} x_{k}\right\|_{T}>4\left\|\sum_{k=1}^{N}\right\| x_{k}\left\|_{T} e_{a_{k}}\right\|_{T}^{\#} .
$$

Let $x=\sum_{k=1}^{N} x_{k}$. Then clearly $\|x\|_{T}>\|x\|_{\infty}$. Hence there exists $n \geq 2$, and finite intervals $n \leq E_{1}<E_{2}<\cdots<E_{n}$ so that $E_{k} x \neq 0$ for $k=1,2, \ldots, n$ and

$$
\|x\|_{T}=\frac{1}{2} \sum_{k=1}^{n}\left\|E_{k} x\right\|_{T}
$$

Using the minimal cardinality of $x$ we have that $\cup_{k=1}^{n} E_{k}$ contains supp $x$. Note first that for any $j$ we have

$$
\left\|x_{j}\right\|_{T} \geq \frac{1}{2} \sum_{k=1}^{n}\left\|E_{k} x_{j}\right\|_{T} .
$$

Now let $G_{k}=\left\{j: \operatorname{supp} x_{j} \subset E_{k}\right\}$ and let $H_{k}$ be the set of $j$ so that $a_{j} \in E_{k}$ but $j \notin G_{k}$.

Then $\left|H_{k}\right|<\min E_{k}$ so that

$$
\left\|\sum_{j \in H_{k}}\right\| x_{j}\left\|_{T} e_{a_{j}}\right\|_{T} \geq \frac{1}{2} \sum_{j \in H_{k}}\left\|x_{j}\right\|_{T} .
$$

Thus

$$
\frac{1}{2} \sum_{j \in H_{k}} \sum_{l=1}^{n}\left\|E_{l} x_{j}\right\|_{T} \leq 2\left\|\sum_{j \in H_{k}}\right\| x_{j}\left\|_{T} e_{a_{j}}\right\|_{T} .
$$

Also, by our minimality assumption we have

$$
\frac{1}{2}\left\|E_{k} \sum_{j \in G_{k}} x_{j}\right\|_{T} \leq 2\left\|\sum_{j \in G_{k}}\right\| x_{j}\left\|_{T} e_{a_{j}}\right\|_{T}^{\#} .
$$

Combining these statements we obtain

$$
\begin{aligned}
\frac{1}{2} \sum_{k=1}^{n}\left\|E_{k} x\right\|_{T} & \leq 2 \sum_{k=1}^{n}\left\|\sum_{j \in G_{k}}\right\| x_{j}\left\|_{T} e_{a_{j}}\right\|_{T}^{\#}+2 \sum_{k=1}^{n}\left\|\sum_{j \in H_{k}}\right\| x_{j}\left\|_{T} e_{a_{j}}\right\|_{T}^{\#} \\
& \leq 4\left\|\sum_{j=1}^{N}\right\| x_{j}\left\|_{T} e_{a_{j}}\right\|_{T}^{\#}
\end{aligned}
$$

as required. 
Proposition 5.12. Tsirelson space $T$ is right-dominant.

Proof. Suppose that $\left(x_{j}\right)_{j=1}^{n},\left(y_{j}\right)_{j=1}^{n}$ are two disjoint sequences with $x_{j}, y_{j} \in c_{00}$ and $\left\|x_{j}\right\|_{T} \leq\left\|y_{j}\right\|_{T}$ and supp $x_{j}<\operatorname{supp} y_{j}$ for $1 \leq j \leq n$. Let $a_{j}=\max \operatorname{supp} x_{j}$. Then

$$
\left\|\sum_{j=1}^{n} x_{j}\right\|_{T} \leq 4\left\|\sum_{j=1}^{n}\right\| x_{j}\left\|_{T} e_{a_{j}}\right\|_{T}^{\#}
$$

The proof of Lemma II.1 of [8] works for the norm \|\|$_{T}^{\#}$ with only notational changes and yields that

$$
\left\|\sum_{j=1}^{n}\right\| x_{j}\left\|_{T} e_{a_{j}}\right\|_{T}^{\#} \leq\left\|\sum_{j=1}^{n} y_{j}\right\|_{T}^{\#}
$$

since $\left\|x_{j}\right\|_{T} \leq\left\|y_{j}\right\|_{T}^{\#}$. Combining we obtain that

$$
\left\|\sum_{j=1}^{n} x_{j}\right\|_{T} \leq 16\left\|\sum_{j=1}^{n} y_{j}\right\|_{T} .
$$

Thus $T$ is right-dominant.

Remark. Of course this implies that $p$-convexified Tsirelson $T^{(p)}$ also is rightdominant for $1<p<\infty$.

Theorem 5.13. Tsirelson space and its dual have unique unconditional bases.

Proof. We have $T$ right-dominant and clearly $r(T)=1$. We need only observe that the canonical basis $\left(e_{n}\right)$ is equivalent to $\left(e_{2 n}\right)$ in $T([8]$ p. 14) and apply Theorem 5.7.

Theorem 5.13 answers a question in [3], where it is shown that convexified Tsirelson $T^{(2)}$ has a unique unconditional basis. In fact much more is true as with $T^{(2)}$ (cf. Theorem 7.9 of [3]). In fact one could prove Theorems 5.13 and 5.14 directly from Theorem 3.5, by using known results, but it seems more natural to invoke the theory of right-dominant bases as here.

Theorem 5.14. Every complemented subspace of $T$ with an unconditional basis has a unique unconditional basis.

Proof. By Theorem 5.7, every complemented normalized unconditional basic sequence is equivalent to a subsequence of the canonical basis. The result follows in the same way as the preceding result, since every subsequence of the basis is right-dominant and equivalent to its square ([8] p. 14). 


\section{Further examples: Orlicz SEQuence SPACES}

In this section we construct some examples of spaces with unique unconditional basis but such that some complemented subspace fails to have unique unconditional basis.

Let $F$ be an Orlicz function satisfying the $\Delta_{2}-$ condition, normalized such that $F(1)=1$. If we set $\phi(\tau)=s F^{\prime}(s) / F(s)$ where $s=e^{-\tau}$ then we can write $F$ in the form

$$
F(t)=\exp \left(-\int_{0}^{\log (1 / t)} \phi(\tau) d \tau\right)
$$

for $0<t \leq 1$. It will be convenient to let $\Phi(u)=\int_{0}^{u} \phi(\tau) d \tau$ for $s \geq 0$.

Lemma 6.1. Suppose $x_{1}, \ldots, x_{n}$ are disjoint in $\ell_{F}$, and satisfy $\left\|x_{k}\right\|_{\ell_{F}}=1$. Suppose $e^{-\tau_{k}}=\left\|x_{k}\right\|_{\infty}$ and let $q_{k}=\sup _{\tau \geq \tau_{k}} \phi(\tau)$ and $r_{k}=\inf _{\tau \geq \tau_{k}} \phi(\tau)$. Then for any $a_{1}, \ldots, a_{n} \in \mathbf{R}$ with $\left\|\sum_{k=1}^{n} a_{k} x_{k}\right\|_{\ell_{F}}=1$ we have

$$
\sum_{k=1}^{n}\left|a_{k}\right|^{q_{k}} \leq 1 \leq \sum_{k=1}^{n}\left|a_{k}\right|^{r_{k}} .
$$

Proof. The proof is essentially trivial. We need only observe that if $j \in \operatorname{supp} x_{k}$,

$$
F\left(\left|a_{k} x_{k}(j)\right|\right)=\exp \left(\Phi\left(\log \left|x_{k}(j)\right|^{-1}\right)-\Phi\left(\log \left|a_{k} x_{k}(j)\right|^{-1}\right) F\left(\left|a_{k} x_{k}(j)\right|\right),\right.
$$

and that, since $\left|a_{k}\right| \leq 1$ and $\left|x_{k}(j)\right| \leq e^{-\tau_{k}}$,

$$
r_{k} \log \left|a_{k}\right|^{-1} \leq \Phi\left(\log \left|a_{k} x_{k}(j)\right|^{-1}\right)-\Phi\left(\log \left|x_{k}(j)\right|^{-1}\right) \leq q_{k} \log \left|a_{k}\right|^{-1} .
$$

Lemma 6.2. If $\lim _{t \rightarrow \infty} \phi(t)=1$ then the Orlicz sequence space $\ell_{F}$ is antiEuclidean.

Proof. Note first that $\ell_{F}$ has cotype 2. Assume that for some $M$ and every $n$ there exist operators $S_{n}: \ell_{2}^{2 n} \rightarrow \ell_{F}$ and $T_{n}: \ell_{F} \rightarrow \ell_{2}^{2 n}$ so that $T_{n} S_{n}$ is the identity on $\ell_{2}^{2 n},\left\|S_{n}\right\|=1$ and $\left\|T_{n}\right\| \leq 1$.

For fixed $n$, we may pick by induction an orthonormal basis $\left(f_{k}\right)_{k=1}^{2 n}$ so that if $v \in\left[f_{k}\right]_{k=j+1}^{2 n}$ then $\left\|S_{n} v\right\|_{\infty} \leq\left\|S_{n} f_{j}\right\|_{\infty}$. Let $H_{n}=\left[f_{k}\right]_{k=n+1}^{2 n}$. Then if $v \in H_{n}$, $\left\|S_{n} v\right\|_{\infty} \leq\left\|S_{n} f_{n+1}\right\|_{\infty}=\alpha_{n}$, say. For fixed $k \in \mathbf{N}$ we have $\sum_{j \in E}\left|S_{n} f_{j}(k)\right| \leq$ $M|E|^{1 / 2}$, when $E \subset[n]$. It follows that if $E_{k}=\left\{j \in[n]:\left|S_{n} f_{j}(k)\right| \geq \alpha_{n}\right\}$ then $\left|E_{k}\right| \leq M^{2} \alpha_{n}^{-2}$. But then

$$
\begin{aligned}
\alpha_{n} F^{-1}\left(M^{2} \alpha_{n}^{-2} n^{-1}\right)^{-1} & \leq\left\|\left(\sum_{j=1}^{n}\left|S f_{j}\right|^{2}\right)^{1 / 2}\right\|_{\ell_{F}} \\
& \leq \sqrt{n} .
\end{aligned}
$$


Hence

$$
F\left(\alpha_{n} / \sqrt{n}\right) \leq M^{2} \alpha_{n}^{-2} n^{-1}
$$

Now if $H_{n}=\left[f_{j}\right]_{j=n+1}^{\infty}$ then $\left\|S_{n} v\right\|_{\infty} \leq \alpha_{n}$ for $v \in H_{n}$. It follows from the equation above that as $n \rightarrow \infty$ we have $\lim \alpha_{n}=0$. Now let $\mathcal{U}$ be a nontrivial ultrafilter on the natural numbers. Consider the ultraproduct $\ell_{\infty}\left(\ell_{F}\right) / c_{0, \mathcal{U}}\left(\ell_{F}\right)$ and the closed subspace thereof $Z_{\mathcal{U}}=Z / c_{0, \mathcal{U}}\left(\ell_{F}\right)$ where $Z$ is the set of sequences $\left(x_{n}\right)$ with $\lim \left\|x_{n}\right\|_{\infty}=0$. Then $Z_{\mathcal{U}}$ must contain a complemented Hilbert space. However $Z$, as a Banach lattice, is an abstract L-space. This follows immediately from Lemma 6.1. Thus we have a contradiction.

The Orlicz space $\ell_{F}$ has a symmetric basis and therefore every sequence of constant coefficient blocks is a complemented unconditional basic sequence. Each such sequence is equivalent to the canonical basis in a modular or Orlicz-Musielak sequence space $\ell_{F}\left[s_{n}\right]$ of all sequences $x$ such that $\sum_{j=1}^{\infty} F_{s_{j}}(|x(j)|)<\infty$ where $F_{s}(t)=F(s t) / F(s)$. Conversely the canonical basis of every such modular sequence space $\ell_{F}\left[s_{n}\right]$ is equivalent a sequence of constant coefficient blocks. If $\left(s_{n}\right)$ fails to converge to 0 then (cf [19], Proposition 3.a.5, p. 117) $\ell_{F}\left[s_{n}\right]$ is isomorphic to $\ell_{F}$. If $\lim _{n \rightarrow \infty} s_{n}=0$ then we can suppose that $\left(s_{n}\right)$ is monotonically decreasing.

Let us say that $F$ is multiplicatively convex or $\mathbf{m}$-convex if it satisfies the condition that $F\left(s^{\theta} t^{1-\theta}\right) \leq F(s)^{\theta} F(t)^{1-\theta}$ whenever $0<s, t, \theta<1$. In this case it is clear that $\Phi$ is concave and that $\phi$ is monotonically decreasing.

Now if $F$ is m-convex and $\left(s_{n}\right)$ is a monotone decreasing sequence it is easy to see that if $\alpha \in c_{00}$ and $\left(r_{k}\right)$ is an increasing sequence of natural numbers so that $r_{k} \geq k$ for all $k$ then $\left\|\sum_{k=1}^{\infty} \alpha_{k} e_{r_{k}}\right\|_{\ell_{F}\left[s_{n}\right]} \geq\left\|\sum_{k=1}^{\infty} \alpha e_{k}\right\|_{\ell_{F}\left[s_{n}\right]}$. Thus there is a weak form of dominance for the canonical basis of $\ell_{F}\left[\tau_{n}\right]$. Based on these observations we can repeat the arguments of Propositions 5.4 and 5.5, which only require this weakened version, to obtain the following:

Lemma 6.3. Suppose $F$ is $m$-convex and that $\left(s_{n}\right)$ is sequence with $0<s_{n} \leq 1$ and $s_{n} \downarrow 0$. The canonical basis $\left(e_{n}\right)$ of $\ell_{F}\left[s_{n}\right]$ is equivalent to its square if and only if $\left(e_{n}\right)$ is equivalent to $\left(e_{2 n}\right)$.

Lemma 6.4. Suppose $F$ is m-convex. Suppose $\left(s_{n}\right)_{n=1}^{\infty}$ is a monotone decreasing sequence with $0<s_{n} \leq 1$, and that $\left(u_{n}\right)$ is a complemented normalized disjoint sequence in $\ell_{F}\left[s_{n}\right]$. Then there is a permutation $\pi$ of $\mathbf{N}$ and a sequence $\left(s_{n}^{\prime}\right)_{n=1}^{\infty}$ 
satisfying $0<s_{n}^{\prime} \leq s_{n}$ and such that $\left(u_{\pi(n)}\right)$ is equivalent to the unit vector basis of $\ell_{F}\left[s_{n}^{\prime}\right]$. If in addition $\lim s_{n}=0$ we may suppose that $\left(s_{n}^{\prime}\right)$ is also decreasing.

Remark. If we take $s_{n}=1$ for all $n$, we obtain the fact that every complemented block basis in $\ell_{F}$ is equivalent to a constant coefficient block basic sequence.

Proof. The proof is standard. Suppose $\left(u_{n}^{*}\right)$ are the dual functionals and that $f_{n}=\left|u_{n}\right|\left|u_{n}^{*}\right|$. Let $r_{n}=\max _{k \in \operatorname{supp} u_{n}} s_{k}$. Pick $s_{n}^{\prime \prime}$ so that if $A_{n}=\left\{k:\left|u_{n}(k)\right| s_{k} \geq\right.$ $\left.s_{n}^{\prime \prime}\right\}$ and $B_{n}=\left\{k:\left|u_{n}(k)\right| s_{k} \leq s_{n}^{\prime \prime}\right\}$ then $\left\|f_{n} e_{A_{n}}\right\|_{1},\left\|f_{n} e_{B_{n}}\right\|_{1} \geq \frac{1}{2}$. Then $\left(u_{n}\right)$ is equivalent to both the sequences $\left(u_{n} e_{A_{n}}\right)$ and $\left(u_{n} e_{B_{n}}\right)$. However, since $\phi$ is monotone decreasing then for $k \in A_{n}$ and any $0 \leq \lambda \leq 1$ we have

$$
\frac{F\left(\lambda s_{n}\left|u_{n}(k)\right|\right)}{F\left(s_{n}\left|u_{n}(k)\right|\right)} \leq \frac{F\left(\lambda s_{n}^{\prime \prime}\right)}{F\left(s_{n}^{\prime \prime}\right)} .
$$

This implies that $\left(u_{n} e_{A_{n}}\right)$ is dominated by the unit vector basis of $\ell_{F}\left[s_{n}^{\prime \prime}\right]$. A similar argument with $B_{n}$ gives that $\left(u_{n}\right)$ is equivalent to $\ell_{F}\left[s_{n}^{\prime \prime}\right]$.

To complete the proof suppose $s>0$ and observe that $\left|\left\{n: s_{n} \geq s\right\}\right| \geq \mid\left\{n: r_{n} \geq\right.$ $s\}|\geq|\left\{n: s_{n}^{\prime \prime} \leq \tau\right\} \mid$ so that we can permute $\left(s_{n}^{\prime \prime}\right)$ to form a sequence $\left(s_{n}^{\prime}\right)$ with the desired properties.

Theorem 6.5. Suppose $F$ is $m$-convex and $\lim _{t \rightarrow \infty} \phi(t)=1$. Suppose $Z$ is a complemented subspace of $\ell_{F}$ with an unconditional basis equivalent to its square, and such that $Z$ is not isomorphic to $\ell_{F}$. Then $Z$ has a unique unconditional basis.

Proof. In fact the given unconditional basis is equivalent to the canonical basis of $\ell_{F}\left[s_{n}\right]$ where $s_{n} \downarrow 0$ and $\left(e_{n}\right)$ is equivalent to $\left(e_{2 n}\right)$. By Lemmas 6.2 and 6.4 and Theorem 3.5 we see that any other unconditional basis is permutatively equivalent to the unit vector basis of $\ell_{F}\left[s_{n}^{\prime}\right]$ where $s_{n}^{\prime} \downarrow 0$ is increasing and $s_{n}^{\prime} \leq s_{n}$. But then, we can similarly find an integer $N$ so that the original basis is permutatively equivalent to a complemented disjoint sequence in the $N$-fold product of this basis. Thus if $\left(e_{n}\right)$ is the original basis there exists a permutation $\pi$ so that $\left(e_{\pi(n)}\right)$ is equivalent to the canonical basis of $\ell_{F}\left[s_{1+[n-1 / N]}^{\prime}\right.$. The argument of Proposition 5.4 again establishes that $\left(e_{\pi(n)}\right)$ is equivalent to $\left(e_{n}\right)$. But now the new basis is equivalent to $\left(e_{N n}\right)$ which is also equivalent to $\left(e_{n}\right)$.

We will specialize to consider functions of the form $F(t) \sim t^{p}|\log t|^{-a}$ where $p \geq 1$ and $a>0$. More precisely let $g(\tau)=\min \left(1, \tau^{-1}\right)$ and let $F^{p, a}$ be the Orlicz function corresponding to $\phi=p+a g$ i.e. $F^{p, a}(t)=t^{p+a}$ for $e^{-1} \leq t \leq 1$ and $F^{p, a}(t)=e^{-p a} t^{p}|\log t|^{-a}$ for $0<t \leq e^{-1}$. These functions are convex and m-convex. 
Now suppose $s_{n} \downarrow 0$. For each $n \in \mathbf{N}$ let $N_{n}$ be the greatest index such that $s_{k} \geq \exp \left(-2^{n}\right)$, and let $N_{0}=0$. Let $E_{n}=\left\{N_{n-1}+1 \leq k \leq N_{n}\right\}$ and $V_{n}=\left[e_{k}: k \in E_{n}\right]$.

Proposition 6.6. Suppose $1 \leq p<\infty$ and $a>0$ are fixed. Let $F=F^{p, a}$. Then if $0<s_{n} \leq 1$ and $s_{n} \downarrow 0$, we have

(1) $\ell_{F}\left[s_{n}\right]=\ell_{p}\left(V_{n}\right)$.

(2) There is a constant $C$ depending only on $p, a$, so that if $x \in V_{n}$, then

$$
C^{-1}\|x\|_{\ell_{G_{n}}} \leq\|x\|_{\ell_{F}\left[s_{n}\right]} \leq C\|x\|_{\ell_{G_{n}}} .
$$

(3) $\ell_{F}\left[s_{n}\right]=\ell_{p}$ as a sequence space if and only if there is a constant $K$ so that $N_{n} \leq \exp \left(K 2^{n}\right)$.

Proof. (1) Notice that if $k \in E_{n}$, then $\sup _{\tau \geq\left|\log s_{k}\right|} \phi(\tau) \leq g\left(2^{n-1}\right) \leq p+$ $a 2^{-(n-1)}$. Now by Lemma 6.1 , if $x \in c_{00}$ we have

$$
\left\|\left(\left\|E_{n} x\right\|_{\ell_{F}\left[s_{n}\right]}\right)\right\|_{\ell_{p+a 2^{n-1}}} \leq\|x\|_{\ell_{F}\left[s_{n}\right]} \leq\left\|\left(\left\|E_{n} x\right\|_{\ell_{F}\left[s_{n}\right]}\right)\right\|_{\ell_{p}}
$$

and by the Simons criterion [25] we obtain (1).

(2) If $k \in E_{n}$, we have $F_{s_{k}}(t) \leq F_{\exp \left(-2^{n}\right)}(t)$ for $0 \leq t \leq 1$. Conversely

$$
F_{\exp \left(-2^{n}\right)}(t) \leq \exp \left(\int_{\log 1 / s_{k}}^{2^{n}} g(\tau) d \tau\right) F_{s_{k}}(t) \leq e F_{s_{k}}(t) .
$$

(3) If $x \in V_{n}$ then by Lemma 6.1 we have

$$
\|x\|_{\ell_{p+a 2^{-}(n-1)}} \leq\|x\|_{\ell_{F}\left[s_{n}\right]} \leq\|x\|_{\ell_{p}}
$$

so that if $N_{n}-N_{n-1} \leq N_{n} \leq \exp \left(K 2^{n}\right)$ each $\left(e_{k}\right)_{k \in E_{n}}$ is uniformly equivalent to the usual basis of $\ell_{p}^{N_{n}-N_{n-1}}$. Conversely note that

$$
\left\|\sum_{k=1}^{N_{n}} e_{k}\right\|_{\ell_{F}\left[s_{m}\right]} \leq N_{n}^{1 /\left(p+a 2^{-n}\right)}
$$

so that the condition is also necessary.

We now give a general criterion for checking permutative equivalence of two bases in these special Orlicz modular spaces. 
Lemma 6.7. Suppose $1 \leq p<\infty$ and $a>0$ are fixed and let $F=F^{p, a}$. Suppose $0<s_{n}, s_{n}^{\prime} \leq 1$ and $s_{n}, s_{n}^{\prime} \downarrow 0$. Suppose the the canonical bases of $\ell_{F}\left[s_{n}\right]$ and $\ell_{F}\left[s_{n}^{\prime}\right]$ are permutatively equivalent. Then there is a constant $K$ so that for every $n \geq 1$ and $k \geq 2$ we have

$$
\left|\log s_{n+k}^{\prime}\right|^{-1} \leq\left|\log s_{n}\right|^{-1}+K(\log k)^{-1}
$$

and

$$
\left|\log s_{n+k}\right|^{-1} \leq\left|\log s_{n}^{\prime}\right|^{-1}+K(\log k)^{-1}
$$

Proof. Let us define

$$
\begin{aligned}
D(n, k) & =\inf _{|A|=n+k} \sup _{\substack{B \subset A \\
|B|=k}}\left\|e_{B}\right\|_{\ell_{F}\left[s_{m}\right]} \\
D^{\prime}(n, k) & =\inf _{|A|=n+k} \sup _{\substack{B \subset A \\
|B|=k}}\left\|e_{B}\right\|_{\ell_{F}\left[s_{m}^{\prime}\right]} .
\end{aligned}
$$

Then there is a constant $C$ so that for all $n, k$ we have $C^{-1} D(n, k) \leq D^{\prime}(n, k) \leq$ $C D(n, k)$. Notice however that

$$
D(n, k)=\left\|\sum_{j=n+1}^{n+k} e_{j}\right\|_{\ell_{F}\left[s_{n}\right]}
$$

and hence if $\tau_{j}=\log 1 / s_{j}$,

$$
k^{1 /\left(p+a g\left(\tau_{n+k}\right)\right)} \leq D(n, k) \leq k^{1 /\left(p+a g\left(\tau_{n}\right)\right)}
$$

and similarly for $D^{\prime}(n, k)$. It follows that

$$
\frac{\log k}{p+a g\left(\tau_{n+k}\right)} \leq \log C+\frac{\log k}{p+a g\left(\tau_{n}^{\prime}\right)}
$$

and this combined with a similar inequality with roles reversed gives the result.

Proposition 6.8. Suppose $0<s_{n} \leq 1$ and $s_{n} \downarrow 0$. The canonical basis of $\ell_{F}\left[s_{n}\right]$ is equivalent to its square if and only if there exists $l \geq 1$ so that $N_{n+l}+$ $\exp \left(2^{n+l}\right) \geq 2 N_{n}$ for all $n \geq 1$.

Proof. From the preceding lemma, we obtain that if the canonical basis is equivalent to its square then,

$$
\log \left|\log s_{2 n}\right| \leq \log \left|\log s_{n}\right|+K(\log n)^{-1}
$$


for some constant $K$. Now suppose $N_{n+l}+\exp \left(2^{n+l}\right) \leq 2 N_{n}$. Then $\log N_{n} \geq$ $2^{n+l}-\log 2 \geq 2^{n+l-1}$ and hence

$$
\left|\log s_{N_{n}}\right|^{-1} \leq\left|\log s_{2 N_{n}}\right|^{-1}+2 K 2^{-n-l} \leq(1+2 K) 2^{-n-l} .
$$

Thus $2^{-n-1} \leq(1+2 K) 2^{-n-l}$ so that $l \leq \log _{2}(2+4 K)$. This implies the given criterion.

For the converse, notice that since the standard $\ell_{p}$ - basis is equivalent to some subsequence of the given basis, the canonical basis is equivalent to the canonical basis of a space $\ell_{F}\left[s_{n}^{\prime}\right]$ where $N_{n}^{\prime}=N_{n}+\left[\exp 2^{n}\right]$. It is then clear that for some fixed $l$ we have $N_{n+l}^{\prime} \leq 2 N_{n}^{\prime}$. This in turn implies that $\left|\log s_{2 n}^{\prime}\right| \leq K\left|\log s_{n}^{\prime}\right|$ for some constant $K$. But then $F_{s_{2 n}^{\prime}}(t) \leq K^{a} F_{s_{n}^{\prime}}(t)$ for $0 \leq t \leq 1$ whence the result.

Theorem 6.9. Suppose $a>0$ and let $F(t) \sim t|\log t|^{-a}$ for $t$ near zero. Let $Z$ be a complemented subspace of $\ell_{F}$ with an unconditional basis $\left(u_{n}\right)$. Suppose $Z$ is not isomorphic to $\ell_{F}$. Then:

(1) If $\left(u_{n}\right)$ is equivalent to its square then $Z$ has a unique unconditional basis.

(2) If every complemented subspace of $Z$ with an unconditional basis also has a unique unconditional basis then $Z$ is isomorphic to $\ell_{1}$.

Remark. By combining Propositions 6.6 and 6.8 , it is clear that we can find $\left(s_{n}\right)$ with $s_{n} \downarrow 0$, so that the canonical basis is equivalent to its square, but $\ell_{F}\left[s_{n}\right]$ is not isomorphic to $\ell_{1}$. Thus Theorem 6.9 answers Problem 11.2 of [3] negatively.

Proof. (1) has already been proved above; it is a special case of Theorem 6.5. For (2) we consider $F=F^{1, a}$ and a sequence $0<s_{n} \leq 1$ with $s_{n} \downarrow 1$. Let $N_{n}, E_{n}$ and $V_{n}$ be defined as before and let $M_{n}=N_{n}-N_{n-1}$. We may suppose, without loss of generality that $s_{k}=\exp \left(-2^{n}\right)$ when $N_{n-1} \leq k \leq N_{n}$, by applying Proposition 6.6 (2). Assume that every subsequence of the canonical basis of $\ell_{F}\left[s_{n}\right]$ spans a space with a unique unconditional basis.

Let $P_{n}=\left[\sqrt{M}_{n}\right]$. We use a result of [15] that since the given basis of each $V_{n}$ is symmetric there is an unconditional basis $\left(u_{k}\right)_{k \in E_{n}}$ of each $V_{n}$ uniformly equivalent to the direct sum of $M_{n}-P_{n}$ members of the given basis and $P_{n}$ constant coefficient vectors of length $P_{n}$.

Now if $\mathcal{N}$ is any infinite subset of $\mathbf{N}$ we can consider the basis $\left(u_{k}\right)_{k \in E_{n}, n \in \mathcal{N}}$ of

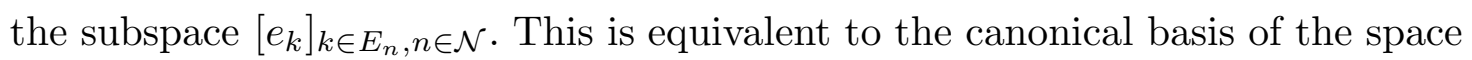

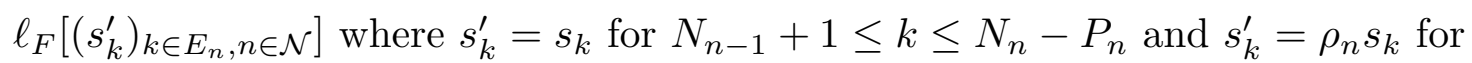
$N_{n}-P_{n}+1 \leq k \leq N_{n}$ where $F\left(\rho_{n} s_{k}\right)=P_{n}^{-1} F\left(s_{k}\right)$. Clearly $\rho_{n} \leq P_{n}^{-(1+a)^{-1}}$. 
Now assume that $\ell_{F}\left[s_{n}\right]$ is not isomorphic to $\ell_{1}$. Then $2^{-n} \log M_{n}$ is unbounded. We then choose $\mathcal{N}=\left\{n_{1}, n_{2}, \ldots\right\}$ inductively so that $\rho_{n_{j}} \exp \left(2^{-n_{j}}\right) \geq$ $\exp \left(2^{-n_{j+1}}\right)$ for $j=1,2, \ldots$ and that $2^{-n_{j}} \log M_{j}$ is unbounded. Then the sequence $\left(s_{k}^{\prime}\right)_{k \in E_{n}, n \in \mathcal{N}}$ is already in decreasing order and the corresponding basis

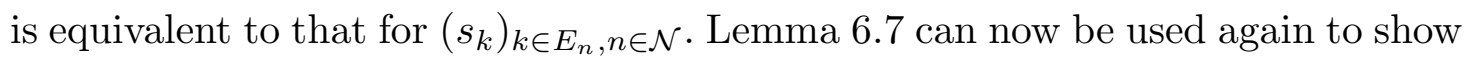
that for some constant $K$ we have that for $n \in \mathcal{N}$,

$$
\left.2^{-n} \leq\left(\left|\log \rho_{n}\right|+2^{n}\right)^{-1}+K\left(\log P_{n}\right)^{-1}\right) .
$$

Since $\left|\log \rho_{n}\right| \geq(1+a)^{-1} \log P_{n}$ this implies that

$$
\log P_{n} \leq K^{\prime} 2^{n}
$$

for some $K^{\prime}$. Thus $\log M_{n} \leq 3 K^{\prime} 2^{n}$ for $n \in \mathcal{N}$ and we have a contradiction.

Remark. This theorem can be proved for wider range of Orlicz functions. Specifically a proof along the same lines can be given if $\phi$ decreases monotonically, $\phi^{\prime}$ is eventually monotone increasing, $\phi(\tau)-1=O\left((\log \tau)^{-1}\right)$ and $\tau(\phi(\tau)-1)$ is eventually increasing.

Finally let us notice it is also possible to give a super-reflexive version.

Theorem 6.10. Let $F(t) \sim t^{2}|\log t|^{-1}$ for $t$ near zero. Let $Z$ be a complemented subspace of $\ell_{F}$ with an unconditional basis $\left(u_{n}\right)$. Suppose $Z$ is not isomorphic to $\ell_{F}$. Then:

(1) If $\left(u_{n}\right)$ is equivalent to its square then $Z$ has a unique unconditional basis.

(2) If every complemented subspace of $Z$ with an unconditional basis also has a unique unconditional basis then $Z$ is isomorphic to $\ell_{2}$.

Proof. The proof of (2) is identical to the proof given above. For (1), we need a result analogous to Theorem 6.5. An inspection of the proof reveals that it is only necessary to show that every complemented unconditional basic sequence is equivalent to a sequence of constant coefficient blocks. It suffices to prove the same result in $\ell_{F}^{*}=\ell_{G}$ where $G(t) \sim t^{2}|\log t|$. But every unconditional basic sequence in $\ell_{G}$ is equivalent to sequence of constant coefficient blocks [6].

Remark. In fact in the dual space $\ell_{G}$ the results hold for any subspace with an unconditional basis (even if uncomplemented).

It may also be shown that the theorem is valid for $F(t) \sim t^{2}|\log t|^{-a}$ where $a \geq 1$.

This requires a complex interpolation technique which we will not expound here. 


\section{REFERENCES}

1. S. Bellenot, Tsirelson superspaces and $\ell_{p}$, J. Functional Analysis 69 (1986), 207-228.

2. B. Bollobas, Combinatorics, Cambridge University Press, 1986.

3. J. Bourgain, P.G. Casazza, J. Lindenstrauss and L. Tzafriri, Banach spaces with a unique unconditional basis, up to a permutation, Memoirs Amer. Math. Soc. No. 322, 1985.

4. J. Bourgain, N.J. Kalton and L. Tzafriri, Geometry of finite-dimensional subspaces and quotients of $L_{p}$, Springer Lecture Notes 1376 (1989), 138-175.

5. P.G. Casazza, W.B. Johnson and L. Tzafriri, On Tsirelson's space, Israel J. Math. 47 (1984), 81-98.

6. P.G. Casazza and N.J. Kalton, Unconditional bases and unconditional finite-dimensional decompositions in Banach spaces, to appear, Israel J. Math.

7. P.G. Casazza, N.J. Kalton and L. Tzafriri, Uniqueness of unconditional and symmetric structures in finite-dimensional spaces, Illinois J. Math. 34 (1990), 793-836.

8. P.G. Casazza and T.J. Schura, Tsirelson's space, Springer Lecture Notes 1363, Springer, 1989.

9. L.E. Dor, On projections in $L_{1}$, Ann. Math. 102 (1975), 463-474.

10. I.S. Edelstein and P. Wojtaszczyk, On projections and unconditional bases in direct sums of Banach spaces, Studia Math. 56 (1976), 263-276.

11. W.T. Gowers, A finite-dimensional normed space with non-equivalent symmetric bases, Israel J. Math. 87 (1994), 143-151.

12. W.T. Gowers, A solution to Banach's hyperplane problem, Bull. London Math. Soc. 26 (1994), 523-530.

13. W.T. Gowers and B. Maurey, Banach spaces with small spaces of operators, preprint.

14. F.L. Hernandez and N.J. Kalton, Subspaces of rearrangement-invariant spaces, to appear.

15. W.B. Johnson, B. Maurey, G. Schechtman and L. Tzafriri, Symmetric structures in Banach spaces, Mem. Amer. Math. Soc. No. 217, 1979.

16. N.J. Kalton, Lattice structures on Banach spaces, Mem. Amer. Math. Soc. No. 493, 1993.

17. G. Köthe and O. Toeplitz, Lineare Raume mit unendlich vielen Koordinaten und Ringen unendlicher Matrizen, J. Reine Angew. Math. 171 (1934), 193-226.

18. J. Lindenstrauss and A. Pełczynski, Absolutely summing operators in $\mathcal{L}_{p}$-spaces and their applications, Studia Math. 29 (1968), 315-349.

19. J. Lindenstrauss and L. Tzafriri, Classical Banach spaces I, Sequence spaces, Springer Verlag, Berlin, Heidelberg, New York, 1977.

20. J. Lindenstrauss and L. Tzafriri, Classical Banach spaces II, Function spaces, Springer Verlag, Berlin, Heidelberg, New York, 1979.

21. J. Lindenstrauss and M. Zippin, Banach spaces with a unique unconditional basis, J. Functional Analysis 3 (1969), 115-125.

22. B.S. Mityagin, Equivalence of bases in Hilbert scales (in Russian), Studia Math. 37 (1970), 111-137.

23. G. Pisier, The volume of convex bodies and geometry of Banach spaces, Cambridge Tracts 94, Cambridge University Press, 1989.

24. C. Schütt, On the uniqueness of symmetric bases in finite dimensional Banach spaces, Israel J. Math. 40 (1981), 97-117.

25. S. Simons, The sequence spaces $\ell\left(p_{\nu}\right)$ and $m\left(p_{\nu}\right)$, Proc. London Math. Soc. (3) 15 (1965), 422-436.

26. P. Wojtaszczyk, Uniqueness of unconditional bases in quasi-Banach spaces with applications to Hardy spaces, II, to appear, Israel J. Math.

27. M. Wojtowicz, On Cantor-Bernstein type theorems in Riesz spaces, Indag. Math. 91 (1988), 93-100.

Department of Mathematics, University of Missouri, Columbia, Mo. 65211 , U.S.A.

E-mail address: pete@math.missouri.edu, mathnjk@mizzou1.missouri.edu 